\title{
CERTAIN TYPES OF INVOLUTORIAL SPACE TRANSFORMATIONS*
}

\author{
BY \\ F. R. SHARPE AND VIRGIL SNYDER
}

\section{INTRODUCTION}

1. Involutorial point transformations of space that have a surface of invariant points can be derived from $(2,1)$ correspondences between two spaces $(x)$ and $\left(x^{\prime}\right)$, namely, the transformation which interchanges the two points in $(x)$ that correspond to the same point in $\left(x^{\prime}\right)$. The correspondence between $(x)$ and $\left(x^{\prime}\right)$ may be expressed algebraically by three equations reducible to the form

$$
\rho x_{i}=\phi_{i}\left(x_{1}, x_{2}, x_{3}, x_{4}\right)=\phi_{i}(x) \quad(i=1,2,3,4) .
$$

In our preceding paper on this subject $\dagger$ it was supposed that one of the equations expressing the correspondence was bilinear in $(x)$ and $\left(x^{\prime}\right)$. This restriction is here removed. The purpose of the present paper is to establish a general method of determining the basis elements of the web of surfaces

$$
\sum_{i=1}^{4} a_{i} \phi_{i}=0
$$

and to enumerate the possible types of associated involutions, when the order of the surfaces of the web is not greater than five.

It is shown that each surface of a web of quartics through a curve of order 8 and genus 2 is invariant under two distinct involutions whose product is discontinuous and of infinite order. These surfaces are similar to, but distinct from, the Fano quartics through a curve of order six and genus 2. Other interesting cases are those in which the surfaces of branch-points $L^{\prime}$ in $\left(x^{\prime}\right)$ are quartics. The focal surface of every line congruence of order two appears among them, and a number of others which together form an uninterrupted chain, having common properties. The surfaces $K$ of coincident points in $(x)$ are birationally equivalent to $L^{\prime}$, and have a different system of interesting properties. When the surfaces of the web are quadrics, $L^{\prime}$ is the

* Presented to the Society, September 3, 1919.

† These Transactions, vol. 20 (1919), pp. 185-202. 
sixteen nodal Kummer surface and $K$ the Weddle surface. This case is well known* but the other cases are believed to be new.

The problem reduces to that of finding a web of surfaces $\sum a_{i} \phi_{i}=0$ having the property that any three surfaces of the web intersect in two variable points. The method has two advantages: first, the involutorial character of the correspondence between the two points $(x)$ is assured; second, certain properties can be more easily discovered in $\left(x^{\prime}\right)$, and then interpreted in $(x)$.

2. General formulas. For convenience of reference the following formulas, due chiefly to Noether $\dagger$ are here collected. If two surfaces $F_{n_{1}}, F_{n_{2}}$ of orders $n_{1}, n_{2}$ contain a curve $C_{m}$ of order $m$ and rank $r$ to multiplicities $i_{1}, i_{2}$, then the residual intersection $C_{\mu}$ meets $C_{m}$ in

$$
\tau=m\left(i_{2} n_{1}+i_{1} n_{2}-2 i_{1} i_{2}\right)-i_{1} i_{2} r
$$

points and has the genus

$$
\mu\left(n_{1}+n_{2}-4\right)-\left(i_{1}+i_{2}-1\right) \tau+1 .
$$

If $F_{n_{1}}, F_{n_{2}}$ have $C_{m}$ to multiplicities $i_{1}, i_{2}$ and also $C_{m^{\prime}}$ to multiplicities $i_{1}^{\prime}, i_{2}^{\prime}$, and $C_{m}$ meets $C_{m^{\prime}}$ in $s$ points, then $C_{\mu}$ meets $C_{m}$ in $\tau-i^{\prime} i^{\prime} s$ points, and $C_{m^{\prime}}$ in $\tau^{\prime}-\left(i_{1} i_{2}^{\prime}+i_{2} i_{1}^{\prime}-i_{1}^{\prime} i_{2}^{\prime}\right) s$ points, provided $i_{1} \geqq i_{1}^{\prime}, i_{2} \geqq i_{2}^{\prime}$.

If $C_{m}$ is $i_{3}$-fold on a third surface $F_{n_{3}}$, then $C_{m}$ is equivalent to $E_{m}$ points of intersection of the three surfaces, where

$$
E_{m}=m\left(i_{2} i_{3} n_{1}+i_{3} i_{1} n_{2}+i_{1} i_{2} n_{3}-2 i_{1} i_{2} i_{3}\right)-r i_{1} i_{2} i_{3} .
$$

The equivalence of $C_{m}$ and $C_{m}$ is

$$
E_{m}+E_{m^{\prime}}-\left(i_{2}^{\prime} i_{3}^{\prime} i_{1}+i_{3}^{\prime} i_{1}^{\prime} i_{2}+i_{1}^{\prime} i_{2}^{\prime} i_{3}-i_{1}^{\prime} i_{2}^{\prime} i_{3}^{\prime}\right) s .
$$

The postulation of $C_{m}, i$-fold on $F_{n}$, is

$$
P_{m}=\frac{i(i+1)}{2}\left(m(n+2)-\frac{2 i+1}{6}(r+2 m)\right) .
$$

For $C_{m}$ and $C_{m^{\prime}}, i$-fold, $i^{\prime}$-fold respectively, the postulation is

$$
P_{m}+P_{m^{\prime}}-\frac{i^{\prime}}{2}\left(i^{\prime}+1\right)\left(3 i-i^{\prime}+1\right) s .
$$

* Hudson, Kummer's Quartic Surface, Chapter XV.

V. Eberhard, Ueber eine räumlich involutorische Verwandtschaft, 7. Grades ..., Breslau Dissertation, 1885.

Snyder, An application of the $(1,2)$ quaternary correspondence to the Kummer and Weddle surfaces, these Transactions, vol. 12 (1911), pp. 354-366.

$\dagger$ Noether, Sulle curve multiple di superficie algebriche, Annali di Mate matica, series 2, vol. 5 (1871), pp. 163-177. 
The postulation of an $l$-fold point through which pass $j$ branches of an $i$-fold curve is

$$
\frac{l}{6}(l+1)(l+2)-\frac{i}{6}(i+1)(3 l-2 i+2) j .
$$

The genus of the variable curve of intersection of two surfaces of the web can also be found from the Riemann-Roch theorem for surfaces. If we assume the surfaces $\phi_{i}(x)=0$ of the web to be regular, we have

$$
r=p_{a}+n-\pi+1
$$

in which $r=2$, the dimensionality of the system of curves on a fixed surface of the system, $p_{a}$ is the arithmetic genus of the surface, $n=2$, the number of points in which two curves of the system intersect (grade of the system), and $\pi$ is the genus of the curve. Hence the

ThEOREM. The genus of a variable curve of intersection of two surfaces of the web is one greater than the arithmetic genus of a general surface of the web.

Moreover the order of $L^{\prime}$, the surface of branch-points in $\left(x^{\prime}\right)$, is $2 \pi+2$. A plane in $(x)$ meets $K$ in a plane curve $\left(s_{1}, K\right)$. The image of $s_{1}$ is a surface $s_{n}^{\prime}$; the image of $K$ is $L^{\prime}$. The image of $\left(s_{1}, K\right)$ is the contact curve of $s_{n}^{\prime}$ and $L^{\prime}$.

The image $s_{N}$ of $s_{1}$ in the involution $I$ in $(x)$ passes through $\left(s_{1} ; K\right)$. The residual intersection of $s_{1}$ with $s_{N}$ has for image a double curve on $s_{n}^{\prime}$. The basis points and fundamental elements will be discussed in connection with each case.

3. Simple basis curve. If the surfaces of the web are of order $n$ and have a simple basis curve $C_{m}$ of genus $p$ and have $\xi$ simple basis points, we have from (3)

and from (5)

$$
(3 n-4) m-2 p+2+\xi=n^{3}-2,
$$

$$
n m-p+1+\xi=\frac{(n+1)(n+2)(n+3)}{6}-4 \text {. }
$$

4. Quadrics. If $n=2$ we have the following cases.
A. $m=0, \xi=6$.
B. $m=1, \xi=2$.
C. $m=2, p=0, \xi=0$.

The case $A$ is the well-known correspondence in which $K$ is the Weddle surface and $L^{\prime}$ the Kummer surface. In case $C$ it can be shown that a line joining conjugate points in $(x)$ passes through a fixed point so that the involution is of the monoidal type already considered by Montesano.*

Case $B$ can be transformed into the special case of $C$ in which the basis conic. consists of two intersecting lines. A similar case exists in which the quadrics have 2 basis points and also touch a fixed plane at a fixed point.

* Montesano, Su le trasformazioni involutorie monoidali, Istit u to Lombardo. R e n d i c o n t i, series 2, vol. 21 (1888), pp. 579-594. 


\section{WeBS OF CUBICS}

5. From the preceding formulas we have

$$
5 m-2 p+2+\xi=25, \quad 3 m-p+1+\xi=16
$$

and find the following possible forms of basis elements.

$A$. Three mutually skew lines $\alpha_{i}$ and four points $P_{i}$.

$B$. Rational quartic curve $\beta_{4}$ and three points $P_{i}$.

C. Quintic curve $\beta_{5}$ of genus 2 and two basis points $P_{i}$.

$D$. Sextic curve $\beta_{6}$ of genus 4 , and one point $P$.

These four cases will be considered in turn.

6. Case A. The image of a plane $s_{1}^{\prime}$ in $\left(x^{\prime}\right)$ is a cubic surface $s_{3}$ in $(x)$, passing through the three lines $\alpha_{i}$ and each of the points $P_{i}$. A line $c_{1}^{\prime}$ of $\left(x^{\prime}\right)$ is transformed into a sextic curve $c_{6}$, the residual intersection of two cubic surfaces of the web. It meets each line $\alpha_{i}$ in 4 points and passes simply through each point $P_{i}$. The image of a point on $\alpha_{i}$ is a straight line in $\left(x^{\prime}\right)$. As the point describes $\alpha_{i}$ the image line describes a rational ruled surface $A_{4}^{\prime}$ of order 4. The images of the points $P_{i}$ are planes $\pi_{i}^{\prime}$. A plane $s_{1}$ has a sextic surface $s_{6}^{\prime}$ for image, and a line $c_{1}$ has a space cubic curve $c_{3}^{\prime}$ for image. A line meeting $\alpha_{i}$ has a fundamental line, generator of $A_{4}^{\prime}$, and a conic for images. The lines meeting $\alpha_{1}, \alpha_{2}, \alpha_{3}$ have points of a curve $\rho^{\prime}$ for images. The curve $\rho^{\prime}$ is therefore the image of the quadric $R_{2}$ determined by $\alpha_{1} ; \alpha_{2}, \alpha_{3}$. Since every plane $s_{1}$ in $(x)$ meets every generator of $R_{2}$ in one point, the image surface $s_{6}^{\prime}$ contains $\rho^{\prime}$ as a simple curve. Moreover, $R_{2}$ meets every cubic surface $s_{3}$ of the web in 3 generators, hence $\rho^{\prime}$ is a space cubic curve. A straight line $c_{1}$ meets $R_{2}$ in 2 points, hence the image cubic $c_{3}^{\prime}$ meets $\rho_{3}^{\prime}$ in 2 points. The image of any line of $R_{2}$ belonging to the same regulus as $\alpha_{i}$ is $\rho_{3}^{\prime}$ itself.

A line through $P_{i}$ has for image a conic and a line in $\pi_{i}^{\prime}$. Through $P_{i}$ passes one line $g_{i, k l}$ meeting $\alpha_{k}, \alpha_{l}$. The image of this line consists of three fundamental lines and a point $G_{i, k l}^{\prime}$. This point lies on all the sextic surfaces $s_{6}^{\prime}$ since $s_{1}$ meets $g_{i, k l}$ in a point. In $(x)$ are 12 such lines $g$ and in $\left(x^{\prime}\right)$ are 12 such points $G^{\prime}$.

An $s_{1}^{\prime}$ through $\rho_{3}^{\prime}$ has for images in $(x)$ the quadric $R_{2}$ and a surface $s_{4}$ through $\alpha_{1}, \alpha_{2}, \alpha_{3}$, having double points at each $P_{i}$. Two quadrics through $\rho_{3}^{\prime}$ meet in a bisecant of $\rho_{3}^{\prime}$. The image quartics $s_{4}$ meet in $\alpha_{1}, \alpha_{2}, \alpha_{3}$, in $c_{4}$ the proper image of $c_{1}^{\prime}$, and in a rational curve $\rho_{9}$ of order 9 , having a triple point at each $P_{i}$, and meeting each $\alpha_{i}$ in 4 points.

In $\left(x^{\prime}\right), L_{4}^{\prime}$ does not contain $\rho_{3}^{\prime}$, but meets it in 6 points of contact. The image of $L_{4}^{\prime}$ is $K_{6}$. The jacobian of the web $\phi_{i}$ consists of $K_{6}$ and of $R_{2}$, hence $K_{6}$ contains each $\alpha_{i}$ to multiplicity 2 , and each point $P_{i}$ to multiplicity 3 . The surfaces $K_{6}, R_{2}$ intersect in $\alpha_{1}, \alpha_{2}, \alpha_{3}$, each taken twice, and in 6 generators, images of the 6 points of contact of $L_{4}^{\prime}$ and $\rho_{3}^{\prime}$. 
Among the cubics $\phi_{i}$ there is one having a double point at $P_{i}$. Moreover, there is a pencil of cubics containing $P_{i} P_{k}$. No nodal cubics are included in this pencil. The image of the line $P_{i} P_{k}$ in $\left(x^{\prime}\right)$ is a straight line. The complete image of this line in $(x)$ consists of $P_{i} P_{k}$ and of a residual $c_{5}$, hence the line in $\left(x^{\prime}\right)$ is a bitangent of $L_{t}^{\prime}$. The line $P_{i} P_{k}$ meets $R_{2}$ in two points, through each of which passes a generator meeting $\alpha_{1}, \alpha_{2}, \alpha_{3}$. Hence these lines lie on every cubic of the pencil containing $P_{i} P_{k}$. The proper residual is a space cubic curve passing through the two remaining basis points and meeting each line $\alpha_{i}$ twice. Since $P_{i} P_{k}$ meets $K_{6}$ in two points, the cubic also passes through these points.

The image plane of a nodal cubic (node at $P_{i}$ ) touches $L_{t}^{\prime}$ along a conic, since its images in $(x)$ consist of $P_{i}$ and the cubic surface. Any line in the plane is a bitangent of $L_{4}^{\prime}$. The images of the line consist of $P_{i}$ and of a rational sextic curve $c_{6}$ having a node at $P_{i}$. The line of intersection of the image planes of two nodal cubics contains two double points on $L_{4}^{\prime}$.

The image in $(x)$ consists of the two points and of two fundamental cubics, each passing through both basis points, and one remaining basis point; the lines $\alpha_{i}$ are bisecants of the cubic curves. The four nodal cubics have for images the planes of the tetrahedron having the double points $\boldsymbol{P}_{i}^{\prime}$ for vertices. Hence through each image cubic curve pass three nodal cubic surfaces. Since $P_{i}^{\prime}$ is on $L_{4}^{\prime}$, the four cubic curves $p_{3}$ are all on $K_{6}$.

The nodal cubics $\psi_{i}$ also contain the lines $g_{i k}$. Their image points $G_{i k}^{\prime}$ are double points on $L_{4}^{\prime}$ and lie on every $s_{6}^{\prime}$ of the system.

These results are expressed by the following Table.

$$
\begin{aligned}
& s_{1}^{\prime} \sim s_{3}: \sum \alpha_{i}+\sum P_{i}, \\
& c_{1}^{\prime} \sim c_{6}, p=1 ; \quad\left[c_{6}, \alpha_{i}\right]=4, \quad\left[c_{6}, P_{i}\right]=1, \\
& \alpha \sim A_{4}^{\prime}: \rho_{3}^{\prime}, \quad P_{i} \sim \pi_{i}^{\prime}, \quad R_{2}: \sum \alpha \sim \rho_{3}^{\prime}, \quad s_{1} \sim s_{6}^{\prime}: \rho_{3}^{\prime}, \quad c_{1} \sim c_{3}^{\prime} ; \quad\left[c_{3}^{\prime}, \rho_{3}^{\prime}\right]=2, \\
& K_{6}: \sum \alpha_{i}^{2}+\sum P_{i}^{3}+12 g_{i}+4 p_{3} \sim L_{4}^{\prime}: 12 G^{\prime 2}+4 P^{\prime 2} .
\end{aligned}
$$

7. The involution $I$. We may now write at once

$$
\begin{aligned}
& s_{1} \sim s_{15}: \sum \alpha_{i}^{5}+\sum P_{i}^{3}+12 g+4 p_{3}^{3}, \\
& \alpha_{i} \sim A_{10}: \alpha_{i}^{4}+2 \alpha^{3}+\sum P_{i}^{4}, \quad R_{2}: \sum \alpha_{i} \sim \rho_{9} .
\end{aligned}
$$

The planes $\alpha_{i} P_{k}$ contains $g_{k, i}$ and $g_{k, l k}$. The image of this plane is $A_{10, i}, \psi_{k}$ and a proper quadric through the other two fundamental lines and the other fundamental points. The plane and the quadric together form a cubic surface of the web. The image plane in $\left(x^{\prime}\right)$ touches $L_{4}^{\prime}$ along a conic; the conic contains 5 points $G_{i}^{\prime}$ and one point $P_{i}^{\prime}$, all double on $L_{i}^{\prime}$. Since each nodal cubic contains three lines $g_{i}$ it follows that the image plane 
contains three points $G_{i}^{\prime}$; it also contains three points $P_{i}^{\prime}$. Hence the surface $L_{4}^{\prime}$ has 16 double points and 16 singular tangent planes; six double points lie in each singular tangent plane and six singular planes pass through each double point. Thus, $L_{4}^{\prime}$ is the Kummer surface. This involution cannot be reduced to that defined by a web of quadrics through six points (Art. 5, Type A). The jacobian of $I$ is made up of the three surfaces $A_{10}$, the quadric $R_{2}$, and the four nodal cubics having nodes at the basis points, each taken twice.

8. Case B. Let the rational quartic curve be $\beta_{4}$, and the three basis points be $P_{1}, P_{2}, P_{3}$. We then have

$$
\begin{aligned}
& s_{\Upsilon}^{\prime} \sim s_{3}: \beta_{4}+\sum P_{i}, \\
& c_{1} \sim c_{5}, p=1 ; \quad\left[c_{5}, \beta_{4}\right]=10, \quad\left[c_{5}, P_{i}\right]=1, \\
& \beta_{4} \sim B_{10}^{\prime}:{\rho^{\prime}}^{3}, \quad R_{2}: \beta_{4} \sim \rho_{2}^{\prime}, \quad s_{1} \sim s_{5}^{\prime}: \rho_{2}^{\prime}+9 G^{\prime} .
\end{aligned}
$$

Through each point $P_{i}$ can be drawn three lines $g$ meeting $\beta_{4}$ in two points. The images of these lines are points $G^{\prime}$. The three nodal cubic surfaces (having nodes at $P_{i}$ ) have a common cubic curve $p_{3}$ whose image is a point $P^{\prime}$, common to the three singular planes, images of the nodal cubics. The residual intersections of the nodal cubics are three fundamental conics $h_{2}$ whose images are points $H^{\prime}$.

There is a $(1,2)$ correspondence of the Geiser type between the plane of $\rho_{2}^{\prime}$ and of $P_{1} P_{2} P_{3}$; the fundamental points are $P_{i}$ and the four points on $\beta_{4}$.

The residual image of $\rho_{2}^{\prime}$ is a plane quartic curve $\rho_{4}$ having a double point at each point $P_{i}$, meeting $\beta_{4}$ in four points, and meeting $R_{2}$ in four other points, all on $K_{6}$; through each passes a fundamental line $t$.

In the involution $I$ the results are

$$
\begin{gathered}
s_{1} \sim s_{12}: \beta_{4}^{4}+\sum P_{i}^{5}+9 g_{i}+(R, P)+4 t+3 h_{2}^{2}+p_{3}^{3}, \\
\beta_{4} \sim B_{24}: \beta_{4}^{3}+\sum P_{i}^{10}, \quad \rho_{4} \sim R_{2}: \beta_{4}, \\
(R, P) \equiv \text { plane section of } R_{2} \text { by } P_{1} P_{2} P_{3} .
\end{gathered}
$$

In every case the image of a basis point is the surface of the web having that point for node. This will be understood in all subsequent cases.

The surface $L_{4}^{\prime}$ has 13 double points and three singular tangent planes. It is the complete focal surface of a line congruence of order 2 and class $5 . *$

* Kummer, Ueber die algebraischen Strahlensysteme, in's Besondere über die der ersten und zweiten Ordnung, A b handlungender k. A kademie der Wissenschaften z u B e r li in, 1866, see pp. 88-94. 
9. Case C. Let the quintic curve be $\beta_{5}$ and the basis points $P_{i}$. Then we may write

$$
\begin{aligned}
& s_{1}^{\prime} \sim s_{3}: \beta_{5}+2 P_{i}, \\
& c_{1}^{\prime} \sim c_{4}, p=1 ; \quad\left[c_{4}, \beta_{5}\right]=8, \quad\left[c_{4}, P_{i}\right]=1, \\
& \beta_{5} \sim B_{8}^{\prime}+\rho_{1}^{\prime 3}, \quad R_{2}: \beta_{5} \sim \rho_{1}^{\prime}, \quad s_{1} \sim s_{4}^{\prime}: \rho_{1}^{\prime}+8 G^{\prime} .
\end{aligned}
$$

The nodal cubics intersect in the two fundamental conics $p_{2}$. The residual image of $\rho_{1}^{\prime}$ is $\rho_{1}=P_{1} P_{2}$. This line meets $R_{2}$ in two points through each of which passes a line $t$ lying on $R_{2}$ and $K_{6}$. The images of these lines in $\left(x^{\prime}\right)$ are the points of contact of $\rho_{1}^{\prime}$ and $L_{4}^{\prime}$.

In $I$ we have

$$
\begin{aligned}
& s_{1} \sim s_{9}: \beta_{5}^{3}+2 P_{i}^{4}+8 g+2 p_{2}^{2}+2 t+\rho_{1}, \\
& \beta_{5} \sim B_{18}: \beta_{5}^{5}+2 P_{i}^{8}, \quad \rho_{1} \sim R_{2}: \beta_{5} .
\end{aligned}
$$

The surface $L_{4}^{\prime}$ has 10 double points and 2 singular planes.

10. Case D. Given any point $A$ in $(x)$. The quadric through $\beta_{6}$ and any plane through $A P$ form a composite cubic of the web. The line $A P$ meets a proper cubic of the web through $A$ in a third point $B$. The points $A, B$, conjugate in the involution $I$, are therefore always collinear with $P$, hence this involution is of the monoidal type, and will not be considered further.

\section{Web of Quartics}

11. Simple basis curves. If the basis curve is simple, the web has no basis points. From the preceding formulas the only condition to be satisfied is

$$
4 m-p=30 \text {. }
$$

If $\beta_{m}$ is not composite, $m=11,10,9$, or 8 . The first of these cases was discussed as Type $I$ in our previous paper.* The others will be designated by $A, B, C$, and discussed in turn, thus:
A. $m=10, p=10$,
B. $m=9, \quad p=6$,
C. $m=8, \quad p=2$.

12. Case A. The image of a plane $s_{1}^{\prime}$ being a surface $s_{4}$ through $\beta_{10}$, it follows that the image of a line $c_{1}^{\prime}$ is a sextic $c_{6}$ of genus 2 meeting $\beta_{10}$ in 22 points. A curve of order $m$ and genus $p$ has

$$
\begin{aligned}
&\left.\frac{((m-1)(m-2)}{6}-2 p\right)((m-1) \\
&(m-2)-2 p-8 m+22) \\
&-\frac{m}{24}(m-2)(m-3)(m-13)
\end{aligned}
$$

${ }^{*}$ L. c. 
quadrisecants. ${ }^{*}$ Hence the curve $\beta_{10}$ has $x=31$ quadrisecants whose images in $\left(x^{\prime}\right)$ are points. The curve may also have conics, cubics $\cdots$ meeting it in $8,12,16 \cdots$ points whose images are also points. Let the numbers of these be $y, z, u \cdots$ respectively. The image of $s_{1}$ in $I$ is $s_{23}$ having $\beta_{10} 6$-fold. Since $I^{\prime}$ is of order 6 , therefore $K$ is of order 12 with $\beta_{10}$ triple. The plane $s_{1}$ meets its image $s_{23}$ in $\left(s_{1}, K_{12}\right)$ and a residual $\delta_{11}$ having the points $\left(s_{1}, \beta_{10}\right)$ triple. The image of $\delta_{11}$ in $\left(x^{\prime}\right)$ is the double curve $\delta^{\prime}$ of $s_{7}^{\prime}$. A fundamental curve of order $k$ meets $s_{1}$ in $k$ points which are simple on $\left(s_{1}, K_{12}\right)$ and $k-1$ fold on $\delta_{11}$.

The genus of $\delta_{11}$ is therefore $15-3 z-12 u-30 v-60 w$. The surface $s_{6}^{\prime}$ being rational it follows that the postulation of $\delta_{7}^{\prime}$ for the adjoint quadrics of $s_{6}^{\prime}$ is 10 . To a fundamental curve of order $k$ corresponds a $(k(k-1) / 2)$ fold point of $\delta_{7}^{\prime}$, hence by formula (7) we have

$$
10=14-p_{\delta_{7}^{\prime}}+1-2 z-8 u-20 v-40 w .
$$

The number of branch points in the $(1,2)$ correspondence between the points of $\delta_{7}^{\prime}$ and $\delta_{11}$ is therefore, by the Zeuthen formula,

$$
\begin{aligned}
\eta & =(30-6 z-24 u-60 v-120 w-2) \\
& =2 z+8 u+20 v+40 w+12 .
\end{aligned}
$$

The 132 intersections of $\delta_{11}$ and $\left(s_{1}, K_{12}\right)$ are made up of 90 on $\beta_{10}$, of a simple intersection at two points for each of the $y$ conics, a double point on $\delta_{11}$ simple on $K_{12}$, at three points for each of the $z$ cubics, etc., and $\eta$ other intersections, hence

$$
2 y+6 z+12 u+20 v+30 w+\eta=42 .
$$

The intersection of $s_{23}$ and $K_{12}$ consists of $\left(s_{1}, K_{12}\right)$, of $\beta_{10}$ taken 6-fold on $s_{23}$, 3 -fold on $K_{12}$, and of fundamental lines, conics, etc., hence

$$
x+4 y+9 z+16 u+25 v+36 w=84 \text {. }
$$

Similarly, considering the intersection of two surfaces $s_{23}$ of the web, we find

$$
x+8 y+27 z+64 u+125 v+216 w=146 .
$$

From these equations we have the only possible solution

$$
x=31, \quad y=11, \quad z=1, \quad \eta=14 \text {. }
$$

We have therefore for the correspondence

$$
\begin{aligned}
& s_{1}^{\prime} \sim s_{4}: \beta_{10}, \quad c_{1}^{\prime} \sim c_{6}, p=2, \quad\left[c_{6}, \beta_{10}\right]=22, \\
& s_{1} \sim s_{6}^{\prime}: 31 G^{\prime}+11{P^{\prime}}^{2}+Q^{\prime 3}, \\
& K_{12}: \beta_{10} \sim L_{6}^{\prime}: 31 G^{\prime 2}+11 P^{\prime 2}+Q^{\prime 2}, \quad \beta_{10} \sim B_{22}^{\prime} .
\end{aligned}
$$

* See Pascal's Repertorium, 1st edition, vol. 2, p. 231. 
In the involution $I$ we have

$$
s_{1} \sim s_{23}: \beta_{10}^{6}+31 g+11 p_{2}^{2}+q_{3}^{3}, \quad \beta_{10} \sim B_{88}: \beta_{10}^{23} .
$$

Each $s_{4}$ of the web is a Fano surface.*

The effect of the involution on curves belonging to a surface of the web may be very easily obtained from the results just given; they lead at once to the theorems given by Severi. $\dagger$

13. Case B. We first find $x=30$ by formula (9), then, proceeding as in Case $A$, we obtain $y=12, z=5$, hence

$s_{1}^{\prime} \sim s_{4}: \beta_{9}, \quad c_{1}^{\prime} \sim c_{7}, p=2 ; \quad\left[c_{7}, \beta_{9}\right]=26, \quad s_{1} \sim s_{7}^{\prime}, \quad \beta_{9} \sim B_{26}^{\prime}$, $K_{12}: \beta_{9}^{3}+30 g+12 p_{2}+5 q_{3} \sim L_{6}^{\prime}: 30 G^{\prime}+12{P^{\prime 2}}^{2}+5 Q^{\prime 3}$.

In $I$ we have

$$
s_{1} \sim s_{27}: \beta_{9}^{7}+30 g+12 p_{2}^{2}+5 q_{3}^{3}, \quad \beta_{9} \sim B_{104}: \beta_{9}^{29} .
$$

14. Case C. Here the characteristics are

$$
\begin{aligned}
& s_{1}^{\prime} \sim s_{4}: \beta_{8}, \quad c_{1}^{\prime} \sim c_{8}, p=2 ; \quad\left[c_{8}, \beta_{8}\right]=30, \\
& \beta_{8} \sim B_{30}^{\prime}, \quad s_{1} \sim s_{8}^{\prime}, \quad K_{12}: \beta_{8}^{3} \sim L_{6}^{\prime} .
\end{aligned}
$$

In $I$ we have

$$
\begin{aligned}
& s_{1} \sim s_{31}: \beta_{8}^{8}, \quad \beta_{8} \sim B_{120}: \beta_{8}^{31}, \\
& x=31, \quad y=10, \quad z=9, \quad u=1 .
\end{aligned}
$$

Each surface of the web of quartics is invariant under $I$. Any two surfaces of the web meet in $\beta_{8}$ and in a curve $c_{8}$ with similar chiracteristics. There exists a web having $c_{8}$ as basis curve, every surface of which is invariant under a second involution $I^{\prime}$. Every surface of the pencil of surfaces containing both $\beta_{8}$ and $c_{8}$ is invariant under both $I$ and $I^{\prime}$, but not point for point. In fact the transformation $I I^{\prime}$ is non-periodic for points on a given surface $F$ of the pencil, as we proceed to prove by the method of Severi. $\neq$ Let $\left|C_{4}\right|$ denote the system of plane sections of $F$. Then

$$
\beta_{8}=4 C_{4}-C_{8} . \quad\left[C_{4}, C_{4}\right]=4, \quad\left[C_{4}, C_{8}\right]=8, \quad\left[C_{8}, C_{8}\right]=2 .
$$

By the involution $I$ a plane is transformed into a surface of order 31 , having $\beta_{8}$ 8-fold while $\left|C_{8}\right|$ remains invariant. Since $\left|C_{4}\right|$ and $\left|C_{8}\right|$ constitute a

* Fano, Sopra alcune superficie del quarto ordine rappresentabili sul piano doppio, $\mathbf{R}$ e $\mathbf{n} \mathrm{d}$ i contidel Reale Istituto Lombardo, series 2, vol. 39 (1906), pp. 1071-1086.

† Severi, Complementi alla teoria della base per la totalità delle curve di una superficie algebrica, Rendiconti del Circolo Matematico di Palermo, vol. 30 (1911), pp. 265-288.

$\ddagger$ L. c. 
base on $F$, the involution $I$, for curves on $F$, is completely expressed by

$$
C_{4} \sim 31 C_{4}-8\left(4 C_{4}-C_{8}\right)=8 C_{8}-C_{4}, \quad C_{8} \sim C_{8} .
$$

Similarly, $I^{\prime}$ is expressed by

$$
C_{4} \sim 8 \beta_{8}-C_{4}, \quad \beta_{8} \sim \beta_{8},
$$

that is, by

$$
C_{4} \sim 31 C_{4}-8 C_{8}, \quad C_{8} \sim 120 C_{4}-31 C_{8} .
$$

Hence, $I I^{\prime}$ is expressed by

$$
C_{4} \sim 929 C_{4}-240 C_{8}, \quad C_{8} \sim 120 C_{4}-31 C_{8},
$$

which is non-periodic. We have therefore proved the following

Theorem. A quartic surface through a general curve of order 8 and genus 2 is invariant under a discontinuous non-periodic group of birational transformations.

15. Case D. In this case $m=7$ and $p=-2$. We find

$$
\begin{aligned}
& s_{1}^{\prime} \sim s_{4}: \beta_{7}, \quad c_{1}^{\prime} \sim c_{9}, p=2 ; \quad\left[c_{9}, \beta_{7}\right]=34, \\
& s_{1} \sim s_{9}^{\prime}, \quad K_{12}: \beta_{7} \sim L_{6}^{\prime} .
\end{aligned}
$$

In the involution $I$ the relation is

$$
s_{1} \sim s_{35}: \beta_{7}^{9} .
$$

From the intersection of $K_{12}$ and $s_{35}$ we obtain the equation

$$
x+4 y+9 z+16 u+25 v+36 w=219
$$

and from the intersection of two surfaces $s_{35}$,

$$
x+8 y+27 z+64 u+125 v+216 w=623 .
$$

The postulation for $s_{35}$ gives

$$
y+4 z+10 u+20 v+35 w=88 .
$$

Two cases are possible. When $\beta_{7}$ consists of a rational quintic $\beta_{5}$ and two lines, $\alpha, \alpha_{1}$, we find $x=31$, made up as follows: first, $\beta_{5}$ has one quadrisecant, by formula (9); the surface of trisecants of $\beta_{5}$ is of order 8 , hence 8 lines meet either line and meet $\beta_{5}$ in three points; finally, the congruence determined by the two lines $\alpha$ has 16 lines in common with that of the bisecants of $\beta_{5}$. These results can also be found by other formulas. ${ }^{*}$ The only possible solution for the remaining unknowns is $y=8, z=10, u=4, v=w=0$. Here $\alpha \sim A_{24}: \beta_{5}^{6}+\alpha_{1}^{6}+\alpha^{7}, \alpha_{1} \sim A_{24}: \beta_{5}^{6}+\alpha_{1}^{7}+\alpha^{6}$, and $\beta_{5} \sim B_{88}: \beta_{7}^{23}$ $+2 \alpha^{22}$.

\footnotetext{
* See Pascal, 1. c., page 231.
} 
In the second case $\beta_{7}$ consists of two space cubics and a line. Here $x=34$, consisting of 12 bisecants of one cubic meeting the line and the other, and of 10 bisecants of both cubics. The only solution now is

$$
y=4, \quad z=16, \quad u=0, \quad v=1, \quad w=0 .
$$

For the line we have

and for each cubic

$$
\alpha \sim A_{24}: \alpha^{7}+2 \beta_{3}^{6} \text {, }
$$

$$
\beta_{3} \sim B_{56}: \alpha^{14}+\beta_{3}^{15}+\beta_{3}^{14} \text {. }
$$

16. Double basis curve. When the web of surfaces of the $(1,2)$ correspondence are of order $n$ and have a common double curve of order $m$ and genus $p$, the equivalence is $(12 n-32) m-16 p+16$ and the postulation is $(3 n-4) m-5 p+5$. If there is also a simple basis curve of order $m^{\prime}$ and genus $p^{\prime}$ meeting the double curve in $s$ points, the additional equivalence is $(3 n-4) m^{\prime}-2 p^{\prime}+2-5 s$ and the additional postulation is $n m^{\prime}-p^{\prime}$ $+1-2 s$.

17. Quartics with a double line a. In this case the preceding formulas become

$$
\begin{gathered}
8 m^{\prime}-2 p^{\prime}+2-5 s+\xi=30, \\
4 m^{\prime}-p^{\prime}+1-2 s+\xi=18,
\end{gathered}
$$

hence $\xi+s=6$ and $4 m^{\prime}-p^{\prime}=11+3 s$, of which the possible solutions are

$\begin{array}{rrrrr} & \xi & s & m^{\prime} & p^{\prime} \\ \mathrm{A} & \mathbf{4} & \mathbf{2} & \mathbf{4} & -1 \\ \mathrm{~B} & \mathbf{3} & \mathbf{3} & \mathbf{5} & \mathbf{0} \\ \mathrm{C} & \mathbf{2} & \mathbf{4} & \mathbf{6} & \mathbf{1} \\ \mathrm{D} & \mathbf{1} & 5 & 7 & 2 \\ \mathrm{E} & 0 & \mathbf{6} & 8 & 3 \\ \mathrm{~F} & \mathbf{0} & \mathbf{6} & 7 & -1\end{array}$

18. Case A. The simple basis quartic consists of two conics $\beta_{2}, \bar{\beta}_{2}$, each meeting $\alpha$ once. Hence

$$
\begin{aligned}
& s_{1}^{\prime} \sim s_{4}: \alpha^{2}+\beta_{2}+\bar{\beta}_{2}+4 P, \\
& c_{1}^{\prime} \sim c_{8}, p=1 ; \quad\left[\alpha, c_{8}\right]=6, \quad\left[\beta_{2}, c_{8}\right]=7, \quad\left[\beta_{2}, c_{8}\right]=7,
\end{aligned}
$$

so that $\alpha \sim A_{6}^{\prime} ; \beta_{2} \sim B_{7}^{\prime} ; \bar{\beta}_{2} \sim \bar{B}_{7}^{\prime}$. The lines meeting $\alpha, \beta_{2}$, and $\bar{\beta}_{2}$ lie on a ruled quartic $R_{4}$ having $\alpha$ triple, which meets a quartic surface of the web in 6 generators whose images are coplanar points on the sextic curve $\rho_{6}^{\prime}$, image of $R_{4}$. The image of a basis point $P$ is a singular tangent plane $\pi^{\prime}$ of $L_{4}^{\prime}$. The complete image in $(x)$ of $\pi^{\prime}$ is the point $P$ and the quartic of the web which has a node at $P$. There are 12 other singular tangent planes of $L_{4}^{\prime}$ corresponding to the composite quartics of the web, one of whose components is either: (1) the plane of the conic $\beta_{2}$ or $\bar{\beta}_{2}$, (2) the plane of $\alpha$ and a 
point $P,(3)$ the quadric through $\alpha, \beta_{2}$ or $\overline{\beta_{2}}$ and two of the points $P$. There are also 16 fundamental curves in $(x)$ whose images are points double on $L_{4}^{\prime}$, namely, 8 lines through the points $P$, meeting $\alpha$ and $\beta_{2}$ or $\bar{\beta}_{2}$, the line of intersection of the planes of $\beta_{2}, \bar{\beta}_{2}$, a quintic curve common to the four nodal quartics and 6 cubic curves, each lying on 3 of the nodal quartics. The existence of the last seven curves follows from the fact that since two quartics of the web meet in a $c_{8}$ of genus 1 , two nodal quartics must meet in a composite $c_{8}$ consisting of a quintic through the points $P$ meeting $\alpha, \beta_{2}$, and $\bar{\beta}_{2}$ each in 4 points, and a cubic through 2 nodes meeting $\alpha$ in 2 points and each conic in 3 points. It is readily verified that in each singular tangent plane of $L_{4}^{\prime}$ are 6 double points. The surface $L_{4}^{\prime}$ is thus the Kummer surface.

The image of $L_{4}^{\prime}$ is $K_{8}: \alpha^{4}+\beta_{2}^{2}+\bar{\beta}_{2}^{2}$ meeting $R_{4}$ in 12 generators $r$, images of the 12 contacts of $\rho_{6}^{\prime}$ with $L_{4}^{\prime}$. The residual image in $(x)$ of $\rho_{6}^{\prime}$ is a curve $\rho_{29}$. This follows from the fact that the image of $s_{1}$ is $s_{8}^{\prime}$ whose residual image in $(x)$ is $s_{27}: \alpha^{13}+\beta_{2}^{7}+\bar{\beta}_{2}^{7}+12 r$, meeting $R_{4}$ in 29 generators whose images in $I$ are the 29 points in which $s_{1}$ meets $\rho_{29}$. A line meeting $\rho_{6}^{\prime}$ has for image a $c_{7}$ meeting $\alpha$ in 5 points and each conic $\beta_{2}$ in 6 points, hence

$$
\alpha \sim A_{6}^{\prime}: \rho_{6}^{\prime} ; \quad \beta_{2} \sim B_{7}^{\prime}: \rho_{6}^{\prime} ; \quad \bar{\beta}_{2} \sim \bar{B}_{7}^{\prime}: \rho_{6}^{\prime} .
$$

The characteristics of the involution $I$ are therefore

$$
\begin{array}{cc}
s_{1} \sim s_{27}: \alpha^{13}+\beta_{2}^{7}+\bar{\beta}_{2}^{7}+4 P^{8}+12 r+\rho_{29}, \\
\alpha \sim A_{20}: \alpha^{10}+\beta_{2}^{5}+\bar{\beta}_{2}^{5}, & \beta_{2} \sim B_{24}: \alpha^{11}+\beta_{2}^{7}+\bar{\beta}_{2}^{6}, \\
\bar{\beta}_{2} \sim \bar{B}_{24}: \alpha^{11}+\beta_{2}^{6}+\bar{\beta}_{2}^{7}, & \rho_{29} \sim R_{4}: \alpha^{3}+\beta_{2}+\bar{\beta}_{2} .
\end{array}
$$

This transformation cannot be reduced to either of the previous ones in which $K$ is equivalent to the Kummer surface.

19. Case $B$. The simple basis curve is now a rational quintic $\beta_{5}$, meeting $\alpha$ in 3 points. The web has three isolated basis points $P$.

so that

$$
\begin{aligned}
& s_{1}^{\prime} \sim s_{4}: \alpha^{2}+\beta_{5}+3 P, \\
& c_{1}^{\prime} \sim c_{7}, p=1 ; \quad\left[\alpha, c_{7}\right]=5, \quad\left[\beta_{5}, c_{7}\right]=13,
\end{aligned}
$$

$$
\alpha \sim A_{5}^{\prime}, \quad \beta_{5} \sim B_{13}^{\prime} .
$$

The bisecants of $\beta_{5}$ which meet $\alpha$ lie on a ruled quartic $R_{4}$ having $\alpha$ triple and meeting a quartic of the web in 5 generators whose images are coplanar points on the quintic image curve $\rho_{5}^{\prime}$ of $R_{4}$. The image of a basis point $P$ is a singular tangent plane $\pi^{\prime}$ of $L_{4}^{\prime}$, whose residual image in $(x)$ is the quartic $\psi$ of the web having a node at $P$. There are 3 other singular tangent planes of $L_{4}^{\prime}$ corresponding to the 3 composite quartics of the web; one of the components is the plane $\pi$ through $\alpha$ and a point $P$. There are 6 lines $g$, two 
through each point $P$, meeting $\alpha$ and $\beta_{5}$. The curve $\beta_{5}$ has a four-fold secant $d$, having a point $D^{\prime}$ for image in $\left(x^{\prime}\right)$. Two composite quartics meet in a conic $\gamma_{i k}$, having a point $\Gamma_{i k}^{\prime}$ for image. Thus

$$
\left[\alpha, \gamma_{12}\right]=1, \quad\left[\beta_{5}, \gamma_{12}\right]=5, \quad\left[P_{3}, \gamma_{12}\right]=1
$$

A composite quartic meets a nodal quartic in a cubic $\beta_{i k}$ having a point $B_{i k}^{\prime}$ for image.

$$
\left[\alpha, \beta_{23}\right]=2, \quad\left[\beta_{5}, \beta_{23}\right]=6, \quad\left[P_{2}, \beta_{23}\right]=\left[P_{3}, \beta_{23}\right]=1 .
$$

Finally, all the nodal quartics pass through a common quartic $\delta$

$$
[\alpha, \delta]=3, \quad\left[\beta_{5}, \delta\right]=7, \quad[P, \delta]=1 .
$$

\begin{tabular}{|c|c|c|c|c|c|c|c|c|c|c|c|c|c|c|}
\hline & $g_{11}$ & $g_{21}$ & $g_{31}$ & $g_{12}$ & $g_{22}$ & $g_{32}$ & $\gamma_{12}$ & $\gamma_{13}$ & $\gamma_{23}$ & $\beta_{23}$ & $\beta_{13}$ & $\beta_{12}$ & $\delta$ & $d$ \\
\hline$f_{1} \pi_{1}$ & $*$ & & & * & & & * & $*$ & & $*$ & & & & * \\
\hline$f_{2} \pi_{2}$ & & * & & & $*$ & & * & & $*$ & & $*$ & & & * \\
\hline$f_{3} \pi_{3}$ & & & * & & & * & & $*$ & $*$ & & & $*$ & & * \\
\hline$\psi_{1}$ & * & & & * & & & & & $*$ & & $*$ & * & * & \\
\hline$\psi_{2}$ & & * & & & * & & & * & & $*$ & & $*$ & * & \\
\hline$\psi_{3}$ & & & * & & & * & $*$ & & & * & * & & * & \\
\hline
\end{tabular}

The relations of the lines and surfaces are represented by the following

Table

The surface $L_{4}^{\prime}$ has 14 double points and 6 singular planes. In each singular plane lie 6 double points, lying on a conic. Through 6 of the double points pass 2 singular planes, and through the remaining 8 pass 3 . The surface is that discussed by Kummer.*

The image of $L_{4}^{\prime}$ is $K_{8}: \alpha^{4}+\beta_{5}^{2}$, meeting $R_{4}$ in 10 generators $r$, images of the 10 contacts of $\rho_{5}^{\prime}$ with $L_{4}^{\prime}$. The residual image of $R_{4}$ is $\rho_{19}$. This follows from the fact that the image of $s_{1}$ is $s_{7}^{\prime}: \rho_{5}^{\prime}$ whose image in $(x)$ is $s_{23}: \alpha^{11}$ $+\beta_{5}^{6}+10 r$; it meets $R_{4}$ in 19 generators, images of the 19 points in which $s_{1}$ meets $\rho_{19}$. Since a line meeting $\rho_{5}^{\prime}$ has for image a sextic meeting $\alpha$ in 4 and $\beta_{5}$ in 11 points, we see that $\alpha \sim A_{5}^{\prime}: \rho_{5}^{\prime}$ and $\beta_{5} \sim B_{13}^{\prime}: \rho_{5}^{\prime 2}$.

In $I$ we have

$$
\begin{gathered}
s_{1} \sim s_{23}: \alpha^{11}+\beta_{5}^{6}+10 r+\rho_{19}, \quad \alpha \sim A_{16}: \alpha^{8}+\beta_{5}^{4}, \\
\beta_{5} \sim B_{44}: \alpha^{20}+_{5}^{12}, \quad P \sim \psi: \alpha^{2}+\beta_{5}+P^{2}, \quad \rho_{19} \sim R_{4}: \alpha^{3}+\beta_{5}, \\
x=7, \quad y=3, \quad z=3, \quad u=1 .
\end{gathered}
$$

* L. c., p. 87. 
20. Case C. In the web are two composite quartics and two nodal ones. There are four lines $g$. The curve $\beta_{6}$ has 2 four-fold secants $d_{1}, d_{2}$ apart irom $\alpha$; they have points $D_{1}^{\prime}, D_{2}^{\prime}$ for images. The 2 nodal quartics intersect in 2 space cubics $\gamma$ having the characteristics

$$
[\alpha, \gamma]=2, \quad\left[\beta_{6}, \gamma\right]=6, \quad[P, \gamma]=1 .
$$

The image of each is a point in $\left(x^{\prime}\right)$.

The nodal quartics intersect the composite quartics in a pair of conics conjugate in $I$ and in the quartic curve $\delta_{4}$ having the scheme

$$
\left[\alpha, \delta_{4}\right]=2, \quad\left[\beta_{6}, \delta_{4}\right]=10, \quad\left[P, \delta_{4}\right]=1 .
$$

The surface $L_{4}^{\prime}$ has 12 singular points and four singular planes; 6 double points lie in each singular plane. It is not a focal surface of any line congruence of order two. We may now write

$$
\begin{aligned}
& s_{1}^{\prime} \sim s_{4}: \alpha^{2}+\beta_{6}+2 P, \quad\left[\alpha, \beta_{6}\right]=4, \\
& c_{1}^{\prime} \sim c_{6} ; \quad p=1 ; \quad\left[c_{6}, \alpha\right]=4, \quad\left[c_{6}, \beta_{6}\right]=12, \\
& R_{4}: \alpha^{3}+\beta_{6} \sim \rho_{4}^{\prime} ; \quad \alpha \sim A_{4}^{\prime}: \rho_{4}^{\prime} ; \quad \beta_{6} \sim B_{12}^{\prime}:{\rho_{4}^{\prime 2},}^{2} \\
& s_{1} \sim s_{6}^{\prime}: \rho_{4}^{\prime}, \quad K_{8}: \alpha^{4}+\beta_{6}^{2}+8 r \sim L_{4}^{\prime}: 12 P^{\prime 2} .
\end{aligned}
$$

In the involution $I$ we have

$$
\begin{aligned}
& s_{1} \sim s_{19}: \alpha^{9}+\beta_{6}^{5}+8 r+\rho_{11}, \quad \alpha \sim A_{12}: \alpha^{6}+\beta_{6}^{3}, \\
& \beta_{6} \sim B_{40}: \alpha^{18}+\beta_{6}^{11}, \quad \rho_{11} \sim R_{4}: \alpha^{3}+\beta_{6}, \quad P \sim \psi, \\
& x=6, \quad y=4, \quad z=2 .
\end{aligned}
$$

21. Case D. We may write at once

$$
\begin{aligned}
& s_{1}^{\prime} \sim s_{4}: \alpha^{2}+\beta_{7}+P, \quad\left[\alpha, \beta_{7}\right]=5, \\
& c_{1}^{\prime} \sim c_{5}, p=1 ; \quad\left[c_{5}, \alpha\right]=3, \quad\left[c_{5}, \beta_{7}\right]=11, \quad\left[c_{5}, P\right]=1, \\
& R_{4}: \alpha^{3}+\beta_{7} \sim \rho_{3}^{\prime} ; \quad \alpha \sim A_{3}^{\prime}: \rho_{3}^{\prime} ; \quad \beta_{7} \sim B_{11}^{\prime}: \rho_{3}^{\prime 2}, \\
& s_{1} \sim s_{5}^{\prime}: \rho_{3}^{\prime} ; \quad K_{8}: \alpha^{4}+\beta_{7}^{2} \sim L_{4}^{\prime}: 10 P^{\prime 2} .
\end{aligned}
$$

In the involution $I$

$$
\begin{aligned}
& s_{1} \sim s_{15}: \alpha^{7}+\beta_{7}^{4}+6 r+\rho_{5}, \\
& \alpha \sim A_{8}: \alpha^{4}+\beta_{7}^{2} ; \quad \beta_{7} \sim B_{36}: \alpha^{15}+\beta^{10} ; \quad \rho_{5} \sim R_{4}: \alpha^{8}+\beta_{7} ; \quad P \sim \psi_{4}, \\
& x=6, \quad y=4 . \text { The lines are } 2 \text { through } P \text { and } 4 \text { quadrisecants of } \beta_{7} . \\
& \text { Trans. Am. Math. Soc. s }
\end{aligned}
$$


22. Case E. Here we have $s_{1}^{\prime} \sim s_{4}: \alpha^{2}+\beta_{8},\left[\alpha, \beta_{8}\right]=6$.

$$
\begin{aligned}
& c_{1}^{\prime} \sim c_{4}, p=1 ; \quad\left[c_{4}, \alpha\right]=2, \quad\left[c_{4}, \beta_{8}\right]=10, \\
& R_{4}: \alpha^{3}+\beta_{8} \sim \rho_{2}^{\prime} ; \quad \alpha \sim A_{2}^{\prime}: \rho_{2}^{\prime} ; \quad \beta_{8} \sim B_{8}^{\prime}:{\rho_{2}^{\prime 2}}^{2}, \\
& s_{1} \sim s_{4}^{\prime}: \rho_{2}^{\prime} ; \quad K_{8}: \alpha^{4}+\beta_{8}^{2} \sim L_{4}^{\prime}: 8 D^{\prime 2} .
\end{aligned}
$$

In $I$ we have

$$
\begin{aligned}
& s_{1} \sim s_{11}: \alpha^{5}+\beta_{8}^{3}+8 d+4 r+\rho_{1}, \\
& \alpha \sim A_{4}: \alpha^{2}+\beta_{8}+8 d ; \quad \beta_{8} \sim B_{32}: \alpha^{14}+\beta_{8}^{9} ; \quad \rho_{1} \sim R_{4}: \alpha^{3}+\beta_{8} .
\end{aligned}
$$

23. Case F. In case $m=7, p=-1$, then $s=6$ and $\xi=0$. The basis curve consists of a rational sextic $\beta_{6}$ which meets $\alpha$ in 5 points, and a line $\bar{\alpha}$ meeting $\alpha$ but not meeting $\beta_{6}$. Through $\beta_{6}$ pass $\infty^{1}$ cubic surfaces, the residual intersection consisting of the four-fold secant $d$ of $\beta_{6}$, and $\alpha$ counted twice.*

In the pencil of cubic surfaces is a ruled cubic $R_{3}$ having $\alpha$ for double directrix, and $d$ for simple directrix. Let $\bar{\alpha}$ be defined by $x_{1}=0, x_{2}=0$, and $\alpha$ by $x_{1}=0, x_{3}=0$. Then if $F=0$ is any cubic of the pencil and $S=0$ any quartic of the web, we may express the correspondence by

and

$$
x_{1}^{\prime}=x_{1} R_{3}, \quad x_{2}^{\prime}=x_{2} R_{3}, \quad x_{3}^{\prime}=x_{1} F, \quad x_{4}^{\prime}=S
$$

Since

$$
s_{1}^{\prime} \sim s_{4}: \alpha^{2}+\bar{\alpha}+\beta_{6} .
$$

we have

$$
\left[\alpha, \beta_{6}\right]=5, \quad[\alpha, \bar{\alpha}]=1, \quad\left[\bar{\alpha}, \beta_{6}\right]=0,
$$

$$
\begin{aligned}
& c_{1}^{\prime} \sim c_{5}, p=1 ; \quad\left[c_{5}, \alpha\right]=2, \quad\left[c_{5}, \bar{\alpha}\right]=3, \quad\left[c_{5}, \beta_{6}\right]=11, \\
& x_{1}=0: \alpha+\bar{\alpha} \sim \rho^{\prime}\left(x_{1}^{\prime}=0, x_{3}^{\prime}=0\right), \\
& R_{3}: \alpha^{2}+\beta_{6} \sim r^{\prime}\left(x_{1}^{\prime}=0, x_{2}^{\prime}=0\right), \quad \beta_{6} \sim B_{11}^{\prime}: \rho^{\prime}+r^{\prime 5}, \\
& \alpha \sim x_{1}^{\prime}=0 \text { counted twice, } \quad \bar{\alpha} \sim \bar{A}_{3}^{\prime}: r^{\prime}+\rho^{\prime}, \quad s_{1} \sim s_{5}^{\prime}: r^{\prime 2}+\rho^{\prime}, \\
& c_{1} \sim c_{4}^{\prime}, p=0 ; \quad\left[c_{4}^{\prime}, r^{\prime}\right]=3, \quad\left[c_{4}^{\prime}, \rho^{\prime}\right]=1 .
\end{aligned}
$$

Let $\bar{\alpha}$ meet $R_{3}$ in $P$, apart from $\alpha$. A plane passed through any generator $h$ of $R_{3}$ and $P$ cuts $R_{3}$ in $h$ and in a conic. The conic passes through $P$, through the point $(h, \alpha)$ and through five points of $\beta_{6}$ not on $h$. Since the image of a point on $\alpha$ is a conic, and of a point on $\beta_{6}$ or $\bar{\alpha}$ is a straight line, it follows that the image of the conic is a point. As $h$ describes $R_{3}$ the image point

* Noether, Zur Grundlegung der Theorie der algebraischen Raumcurven, A b h a n d l u n g e $\mathrm{n}$ der königlich Preussischen Akademie der Wissenschaftenzu Berlin vom Jahre 1882. See page 86. Case $a_{0}^{\prime}$. 
of the associated conic describes $r^{\prime}$. The image of a plane $x_{1}^{\prime}=\tau x_{2}^{\prime}$ through $r^{\prime}$ consists of $R_{3}$ and of the plane $x_{1}=\tau x_{2}$ through $\bar{\alpha}$. The complete image of the latter plane consists of $A_{3}^{\prime}$ and of the plane $x_{1}^{\prime}=\tau x_{2}^{\prime}$ taken twice. A straight line in $x_{1}^{\prime}=\tau x_{2}^{\prime}$ has for image the intersection of the plane $x_{1}=\tau x_{2}$ and a general quartic $s$ of the web. It consists of the fundamental line $\bar{\alpha}$ and of a general cubic through the point on $\alpha$ and the six points on $\beta_{6}$. Hence the $(1,2)$ correspondence between the two planes is of the Geiser type, having the points on $\beta_{6}$ and on $\alpha$ for fundamental points. The line in $\left(x^{\prime}\right)$ meets $r^{\prime}$ in a point whose image is a conic, intersection of $R_{3}$ and $S$. The plane $x_{1}=\tau x_{2}$ meets the conic in $P$ and in one other point lying on the nodal cubic curve in which the plane meets $R_{3}$. This nodal cubic is the image of the point on $\alpha$ in the involution $I$. As $\tau$ varies, the nodal cubic describes $R_{3}$, hence we see that the image of $R_{3}$ in $I$ is the point $(\alpha, \bar{\alpha})$.

Let $g_{1}, g_{2}$ be the two generators of $R_{3}$ through $(\alpha, \bar{\alpha})$, and let $\tau_{i}$ define the plane of the pencil $x_{1}=\tau x_{2}$ through $g_{i}$. The nodal cubic now consists of the conic and $g_{i}$. In a general plane the jacobian of the net of cubics, a sextic having double points at all seven basis points, is the partial section of $K_{8}$ by the plane. In the plane $\tau_{i}$ the conic is part of the jacobian, hence the two conics lie on $K_{8}^{\prime}$. Since the image of $r^{\prime}$ is composite, we conclude that $r^{\prime}$ is a bitangent of $L_{4}^{\prime}$, the poinis of tangency being at the image points of the two conics on $K_{8}$.

A rational $\beta_{6}$ has a ruled surface of trisecants of order 20. Since $\alpha$ is a five-fold secant, it counts for 10 trisecants, hence $\bar{\alpha}$ meets 10 others. In the planes of the pencil $x_{1}=\tau x_{2}$ these lines lie on the jacobians of the net of cubic curves, hence these 10 lines all lie on $K_{8}$.

The plane $x_{1}=0$ meets $\beta_{6}$ in one point $H$ not on $\alpha$. A line through $H$ in this plane goes into a point of $\rho^{\prime}$. The lines $H P$ and $d$ constitute a composite conic on $R_{3}$, the image of which is the point $(0,0,0,1)$ in $\left(x^{\prime}\right)$. The two lines of the pencil $H$ which lie on $K_{8}$ have for images the points of contact of $L_{4}^{\prime}$ and $\rho^{\prime}$. To the points $(\lambda)$ of any line $g$ through $H$ correspond the elements in the plane $x_{1}^{\prime}=\lambda x_{3}^{\prime}$ through $G^{\prime}$.

In $I$ we now have

$$
s_{1} \sim s_{12}: \alpha^{5}+\bar{\alpha}^{4}+\beta_{6}^{3} .
$$

24. The fundamental line $\alpha$. To obtain the images of the points of $\alpha$, first consider the straight lines meeting it. In the equations $x_{1}^{\prime}=x_{1} R_{3}$, etc., replace $x_{1}$ by $k x_{3}, x_{2}$ by $\mu x_{4}$ and divide by $x_{3}^{2}$. Then replace $x_{3}$ by 0 to obtain the image of the point $(0, \mu, 0,1)$. The resulting equations have the form $x_{1}^{\prime}=0, x_{2}^{\prime}=\mu(k, \mu), x_{3}^{\prime}=k(k, \mu), x_{+}^{\prime}=(k, \mu)$, all the second members being non-homogeneous polynomials of order 2 in $k$ and in $\mu$. By eliminating $k$, we have a conic containing the parameter $\mu$ to degree 8 , of 
which 6 roots (intersections with $\beta_{6}$ and with $\bar{\alpha}$ ) are constant, hence there results a quadratic system of conics

$$
\mu^{2} C^{\prime}+\mu \bar{C}^{\prime}+x_{2}^{\prime} u^{\prime}=0,
$$

having the section of $L_{4}^{\prime}$ by the plane $x_{1}^{\prime}=0$ for envelope. Similarly, by eliminating $\mu$ we obtain the quadratic system

$$
k^{2} C_{1}^{\prime}+k \bar{C}_{1}^{\prime}+x_{3}^{\prime} u^{\prime}=0 \text {. }
$$

Every direction in the pencil determined by $\mu$ and $k$ has the same image point in $\left(x^{\prime}\right)$.

By regarding $\mu, k$ as non-homogeneous poin $i$ coördinates in an auxiliary plane $\pi$, the preceding equations define a $(1,2)$ correspondence of the Geiser type between $x_{1}^{\prime}=0$ and $\pi$.

Now let a plane $s_{1}$ meet $\alpha$ in $\mu_{1}$ and another plane $s_{2}$ meet $\alpha$ in $\mu_{2}$. The conics $c_{2}^{\prime}\left(\mu_{1}\right), c_{2}^{\prime}\left(\mu_{2}\right)$ meet in four points, each of which has two images $(\mu, k)$ on $\alpha$. Let them be $\left(\mu_{1}, k_{11}\right),\left(\mu_{1}, k_{21}\right),\left(\mu_{1}, k_{31}\right),\left(\mu_{1}, k_{41}\right)$ and $\left(\mu_{2}, k_{12}\right),\left(\mu_{2}, k_{22}\right),\left(\mu_{2}, k_{32}\right),\left(\mu_{2}, k_{42}\right)$. These directions define 4 tangent planes to the image $s_{12}$ of $s_{2}$ at $\mu_{1}$ and of the image $s_{12}$ of $s_{1}$ at $\mu_{2}$ respectively.

To obtain the tangent of the fifth branch of any $s_{12}$ at $\mu$, consider the section of $s_{1}$ with its own image $s_{12}$. It consists of $\left(s_{1}, K_{8}\right)$ and a residual $c_{4}$ in $(2,1)$ correspondence with the points of the double cubic curve $\delta^{\prime}$ of $s_{5}^{\prime}$. The double cubic meets $r^{\prime}$ in the points of contact of $L_{4}^{\prime}$ and $r^{\prime}$, images of the two conics $\left(R_{3}, K_{8}\right)$ and meets $\rho^{\prime}$ in a variable point $M_{2}^{\prime}$.

The curve $c_{4}$ has a double point at $T$, where $s_{1}$ meets $\bar{\alpha}$. We have seen that every plane $x_{1}=\tau x_{2}$ through $\bar{\alpha}$ is invariant in $I$. It meets $s_{1}$ in a line whose image is a curve of order 8 , meeting it in 6 points on $K_{8}$ and in a pair of conjugate points in $I$. As $\tau$ varies, these conjugate points describe $c_{4}$. When $\tau=0$, the points are $\mu$ and a definite point $M$. The line $M H$ is on $s_{12}$, and forms, with fundamental lines, the complete intersection $x_{1}=0, s_{12}$.

The conic $c_{2}^{\prime}(\mu)$ meets $x_{3}^{\prime}=0(k=0)$ in two points $M_{1}^{\prime}=(\mu, 0)$ and $(\bar{\mu}, k)$ and $M_{2}^{\prime}=\left(\mu, k_{1}\right)^{\prime}$ and $\left(\mu_{1}, 0\right)$. The double curve $\delta^{\prime}$ passes through $M_{2}^{\prime}$ and its tangent fixes $\lambda$. The direction of the tangent to $c_{4}$ at $\mu$ is in the plane $k_{1}$.

Now consider any point $I$ in $x_{1}=0$. Draw the line $D H$, and call the point in which it meets $\alpha$ by its parameter $\mu$. The conic $c_{2}^{\prime}(\mu)$ defined by this point meets $\rho^{\prime}$ in $D_{1}^{\prime}$ and $D_{2}^{\prime}$. The value of $k$ defined by $D_{2}^{\prime}$ is therefore independent of $\lambda$ on the line $D H$. Any plane $s_{1}$ meets $x_{1}=0$ in a line having a point on every line $g$ through $H$. The image of this point is a definite element of $\alpha$, hence the following

THEOREM. All the surfaces $s_{12}$ have a common tangent plane at every point of $\alpha$. 
The table for $I$ may now be written as follows:

$s_{1} \sim s_{12}: \alpha^{5}$ (one branch fixed) $+\bar{\alpha}^{4}+\beta_{6}^{3}, \quad \alpha \sim\left(x_{1}=0\right.$ taken twice), $\bar{\alpha} \sim \bar{A}_{8}: \alpha^{3}+\bar{\alpha}^{3}+\beta_{6}^{2}, \quad \beta_{6} \sim B_{28}: \alpha^{11}+\bar{\alpha}^{10}+\beta_{6}^{7}, \quad(\alpha, \bar{\alpha}) \sim R_{3}$, $x=10+$ two lines $x_{1}=0$ on $K_{8}, \quad y=1+$ two conics of $R_{3}$ on $K_{8}$.

\section{WeB OF QUINTICS}

25. Simple basis curve impossible. For a simple basis curve of order $m$ and genus $p$ we require

$$
11 m-2 p+2+\xi=123, \quad 5 m-p+1+\xi=52 .
$$

Hence $m-\xi=19,6 m-p=70$. If $\xi=0$, then $m=19$, so that the residual intersection of two quintics of the web is a sextic curve of genus five, which is impossible; if $\xi=1$, the residual is a quintic of genus five, which is also impossible, and so on.

26. Double basis line $a$ and simple basis curve $\beta$ of order $m^{\prime}$ and genus $p^{\prime}$. Here we have

$$
11 m^{\prime}-2 p^{\prime}+2-5 s+\xi=79, \quad 5 m^{\prime}-p^{\prime}+1-2 s+\xi=36,
$$

so that $p^{\prime}+3 s=6 m^{\prime}-42$, and $s+\xi=m^{\prime}-7$.

The variable curve of intersection of two surfaces of the web is of genus 3 . Pass a plane through $\alpha$. It meets the surfaces of the web in cubic curves, any two of which meet in 9 points of which $m-s$ are on $\beta_{m}$, and therefore $9-m+s$ on the variable curve of intersection. Thus $m-s \leqq 7$, and consequently $\xi=0$. The variable curve meets $\alpha$ in $12-s$ points, and meets $\beta_{7+s}$ in $44-3 s$ points.

A surface $\Delta_{4}$ contains $\alpha$ and passes through $\beta_{7+8}$. The image of $\Delta_{4}$ is a straight line $\delta^{\prime}$ in $\left(x^{\prime}\right)$. We may now write

$$
\begin{aligned}
& s_{1}^{\prime} \sim s_{5}: \alpha^{2}+\beta_{7+8} ; \quad\left[\alpha, \beta_{7+s}\right]=s ; \quad p \text { of } \beta_{7+8}=3 s, \\
& c_{1}^{\prime} \sim c_{14-8}, \quad p=3 ; \quad\left[\alpha, c_{14-8}\right]=12-s, \quad\left[\beta_{7+8}, c_{14-8}\right]=44-3 s, \\
& \Delta_{4}: \alpha+\beta_{7+8} \sim \delta^{\prime}, \quad s_{1} \sim s_{14-8}^{\prime}: \delta^{\prime 1-s}, \quad \alpha \sim A_{12-8}^{\prime}:{\rho^{\prime-8}}^{9-8} ; \\
& \beta_{7+8} \sim B_{44-3 s}^{\prime}: \delta^{\prime 37-3 s}, \quad K_{12}: \alpha^{6}+\beta_{7+8}^{2} \sim L_{8}^{\prime}: \delta^{\prime 4},
\end{aligned}
$$

and in the involution $I$

$$
\begin{aligned}
& s_{1} \sim s_{25-8}: \alpha^{17-8}+\beta_{7+s}^{3}, \\
& \alpha \sim A_{44-8}: \alpha^{16-8}+\beta_{7+s}^{3} ; \quad 、 \beta_{7+s} \sim B_{67-3 s}: \alpha^{46-3 s}+\beta_{7+s}^{8} .
\end{aligned}
$$


The range of variation of $s$ is $0 \leqq s \leqq 8$, hence there are nine distinct cases of involutions defined by quintics having a double line.*

27. Quintics with a double conic. From Article 16 we have

$$
p^{\prime}+3 s=6 m^{\prime}-25, \quad s+\xi=m^{\prime}-1 .
$$

The only possible solutions are $\xi=0$, and $7 \leqq m^{\prime} \leqq 12$. When $m^{\prime}=7$, $\beta_{7}$ is composite and two types appear, each having $p^{\prime}=-1$. In the first, $\beta_{7}=\beta_{6}+\alpha$, the sextic being rational and not meeting the line $\alpha$. In the second case, $\beta_{7}=\beta_{4}+\beta_{3}$, both rational. We have for all these cases (writing $m$ instead of $m^{\prime}$ )

$$
\begin{aligned}
& s_{1}^{\prime} \sim s_{5}: \gamma_{2}^{2}+\beta_{m} ; \quad\left[\beta_{m}, \gamma_{2}\right]=m-1, \quad p \text { of } \beta_{m} \text { is } 3 m-22, \\
& c_{1}^{\prime} \sim c_{17-m}, \quad p=2 ; \quad\left[c_{17-m}, \gamma_{2}\right]=17-m, \quad\left[c_{17-m}, \beta_{m}\right]=49-3 m, \\
& \gamma_{2} \sim \Gamma_{17-m}^{\prime}: \rho^{\prime 2} ; \quad R_{1}: \gamma_{2} \sim \rho^{\prime} ; \quad \beta_{m} \sim B_{49-3 m}^{\prime}: \rho^{\prime}, \\
& s_{1} \sim s_{17-m}^{1}: \rho^{\prime} ; \quad K_{15}: \gamma_{2}^{6}+\beta_{m}^{3}+3 r \sim L_{6}^{3} .
\end{aligned}
$$

In the involution $I$ we have

$$
\begin{aligned}
& s_{1} \sim s_{83-5 m}: \gamma_{3}^{33-2 m}+\beta^{17-m}+3 r+. \rho_{14-m}, \\
& \gamma_{2} \sim \Gamma_{83-5 m}: \gamma_{2}^{33-2 m}+\beta_{m}^{17-m} ; \quad \beta_{m} \sim B_{244-15 m}: \gamma_{2}^{97-6 m}+\beta_{m}^{50-3 m}, \\
& \rho_{14-m} \sim R_{1}: \gamma_{2} ; \quad x=\left(m^{3}-24 m^{2}+197 m-402\right) / 6 .
\end{aligned}
$$

28. Quintics with two non-intersecting double lines. The only possible case is that in which the residual basis curve $\beta_{m}$ of order $m$ is of genus 4; there are $11-m$ isolated basis points. The fundamental lines $\alpha$ and $\bar{\alpha}$, $\beta_{m}$ lie on a ruled surface $R_{6}$ of order 6 , genus 4 . We now have, for $7 \leqq m \leqq 11$,

$$
\begin{aligned}
& s_{1}^{\prime} \sim s_{5}: \alpha^{2}+\bar{\alpha}^{2}+\beta_{m}+(11-m) P ; \quad p \text { of } \beta_{m} \text { is 4; } \\
& {\left[\alpha, \beta_{m}\right]=\left[\bar{\alpha}, \beta_{m}\right]=m-3 \text {, }} \\
& c_{1}^{\prime} \sim c_{17-m}, \quad p=1 ; \quad\left[c_{17-m}, \alpha\right]=\left[c_{17-m}, \bar{\alpha}\right]=15-m \text {, } \\
& {\left[c_{17-m}, \beta_{m}\right]=12 \text {, }} \\
& \alpha \sim A_{15-m}^{\prime}: \rho_{18-m}^{\prime} ; \quad \bar{\alpha} \sim \bar{A}_{15-m}: \rho_{18-m}^{\prime} ; \quad R_{6}: \alpha^{3}+\bar{\alpha}^{3}+\beta_{m} \sim \rho_{18-m}^{\prime} \text {, } \\
& \beta_{m} \sim B_{12}^{\prime}: \rho_{18-m}^{\prime} ; \quad s_{1} \sim s_{17-m}^{\prime}: \rho_{18-m}^{\prime} \text {, } \\
& K_{10}: \alpha^{4}+\bar{\alpha}^{4}+\beta_{m}^{2}+(36-2 m) r+(11-m) P^{2} \sim L_{4}^{\prime},
\end{aligned}
$$

* The webs of quintics having a double line can be generalized immediately to surfaces of order $n$ having $\alpha$ to multiplicity $n-3$. The general involution is

$$
s_{1} \sim s_{9 n-8-20}: \alpha^{9 n-s-28}+\beta_{7+?}^{3} .
$$

No new fundamental elements appear. 
and in the involution $I$

$$
\begin{aligned}
& s_{1} \sim s_{78-5 m}: \alpha^{31-2 m}+\bar{\alpha}^{31-2 m}+\beta^{16-m}, \\
& \alpha \sim A_{69-5 m}: \alpha^{28-2 m}+\bar{\alpha}^{27-2 m}+\beta^{14-m} ; \quad \bar{\alpha} \sim A_{69-5 m}: \alpha^{27-2 m}+\bar{\alpha}^{28-2 m}, \text { etc. } \\
& \beta_{m} \sim B_{54}: \alpha^{21}+\bar{\alpha}^{21}+\beta_{m}^{12}, \quad \rho \sim R_{6}: \alpha^{3}+\bar{\alpha}^{3}+\beta_{m} .
\end{aligned}
$$

The order of $\rho$ is $m^{2}-34 m+282$.

29. Double space cubic $\gamma_{3}$. The simple curve $\beta_{m^{\prime}}$ satisfies the conditions

$$
\begin{aligned}
11 m^{\prime}-2 p^{\prime}+2-5 s+\xi & =23, \\
5 m^{\prime}-p^{\prime}+1-2 s+\xi & =14,
\end{aligned}
$$

so that $p^{\prime}+3 s=6 m^{\prime}-8, s+\xi=m^{\prime}+5$. The following cases exist.

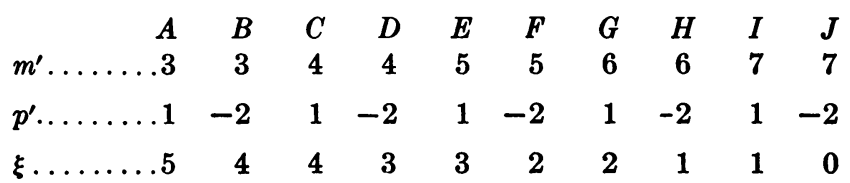

30. Case A. This case is reducible to Case $A$ of Article 5.

31. Case B. The simple cubic consists of two bisecants $\alpha, \bar{\alpha}$ of $\gamma_{3}$, and a line $\beta$ not meeting $\gamma_{3}$. Hence

$$
\begin{aligned}
& s_{1}^{\prime} \sim s_{5}: \gamma_{3}^{2}+\alpha+\bar{\alpha}+\beta+4 P, \\
& c_{1}^{\prime} \sim c_{10}, \quad p=1 ; \quad\left[c_{10}, \gamma_{3}\right]=16, \quad\left[c_{10}, \alpha\right]=2, \quad\left[c_{10}, \beta\right]=8 .
\end{aligned}
$$

The bisecants of $\gamma_{3}$ which meet $\beta$ lie on $R_{4}: \gamma_{3}^{2}+\beta$ and have for images in $\left(x^{\prime}\right)$ the points of a curve $\rho_{7}^{\prime}$. The quadric $H_{2}$ through $\gamma_{3}, \alpha, \bar{\alpha}$ and a cubic through $\gamma_{3}, \beta$, and the points $P$ form a composite quintic of the web. The image of $\mathrm{H}_{2}$ is therefore a line $h^{\prime}$ and the complete image of $h^{\prime}$ consists of $H_{2}$ and a quintic curve $h_{5}$ common to the cubics of the pencil. The quadric $H_{2}$ meets any $s_{5}$ of the web in a conic, the partial image of a point of $h^{\prime}$. The conic meets $\gamma_{3}$ in 3 points, $\alpha$ and $\bar{\alpha}$ each in one, and $\beta$ in 2 points. The surface $L_{4}^{\prime}$ has 16 singular tangent planes, images of the 8 composite quintics containing a quadric through $\gamma_{3}, \alpha$ or $\bar{\alpha}$ and one of the points $P$, the 4 containing a plane through $\beta$ and a point $P$, and the 4 nodal quintics. Two nodal quintics intersect in two quintic curves $t_{5}$. Through each point $P$ passes a bisecant $g$ of $\gamma_{3}$ and a conic $p_{2}$ meeting $\beta$ twice, $\gamma_{3}$ three times, and $\alpha$ or $\bar{\alpha}$. There are also two lines $d$ meeting $\gamma_{3}, \alpha, \bar{\alpha}$, and $\beta$. The four quintics $t_{5}$, the 8 lines $g$, the 8 conics $p_{2}$ and the 2 lines $d$ have for images the 16 double points of $L_{4}^{\prime}$.

The line $h^{\prime}$ is bitangent to $L_{4}^{\prime}$, the points of contact being $D_{i}^{\prime}$, whose images are conics lying on $K_{10}$ and $H_{2}$. The curve $\rho_{7}^{\prime}$ is tangent to $L_{4}^{\prime}$ at 14 points $R^{\prime}$, images of 14 generators $r$ in which $K_{10}$ meets $R_{4}$. 
We may now write

$$
\begin{aligned}
& \alpha \sim A_{2}^{\prime}: h^{\prime}, \quad \bar{\alpha} \sim \bar{A}_{2}^{\prime}: h_{2}^{\prime}, \quad \beta \sim B_{8}^{\prime}: \rho_{7}^{\prime}+h^{\prime 2}, \quad \gamma_{3} \sim \Gamma_{16}^{\prime}: \rho_{7}^{\prime 2}+h^{\prime 3}, \\
& s_{1} \sim s_{10}^{\prime}: h^{\prime 2}+\rho_{7}^{\prime}, \quad c_{1} \sim c_{3}^{\prime}, \quad p=0 ; \quad\left[c_{3}^{\prime}, h^{\prime}\right]=2, \quad\left[c_{5}^{\prime}, \rho_{7}^{\prime}\right]=4, \\
& K_{10}: \gamma_{3}^{4}+\alpha^{2}+\bar{\alpha}^{2}+\beta^{2} \sim L_{4}^{\prime} .
\end{aligned}
$$

and in the involution $I$

$$
\begin{aligned}
& s_{1} \sim s_{41}: \gamma_{3}^{16}+\alpha^{8}+\bar{\alpha}^{8}+\beta^{9}, \\
& \alpha \sim A_{8}: \gamma_{3}^{3}+\alpha^{2}+\bar{\alpha}+\beta^{2}, \quad \bar{\alpha} \sim \bar{A}_{8}: \gamma_{3}^{3}+\alpha+\bar{\alpha}^{2}+\beta^{2}, \\
& \beta \sim B_{32}: \gamma_{3}^{12}+\alpha^{6}+\bar{\alpha}^{6}+\beta^{8}, \quad \gamma_{3} \sim \Gamma_{66}: \gamma_{3}^{26}+\alpha^{13}+\bar{\alpha}^{13}+\beta^{14}, \\
& h_{5} \sim H_{2}: \gamma_{3}+\alpha+\bar{\alpha}, \quad \rho_{45} \sim R_{4}: \gamma_{3}^{2}+\beta .
\end{aligned}
$$

Each point $P$ goes into the quintic of the web having a node at $P$.

32. Case C. Here we have a quartic curve $\beta_{4}, p=1,\left[\beta_{4}, \gamma_{3}\right]=5$, and 4 basis points $P$, hence we may write

$$
\begin{aligned}
& s_{1}^{\prime} \sim s_{5}: \gamma_{3}^{2}+\beta_{4}+4 P, \\
& c_{1} \sim c_{9}, \quad p=1 ; \quad\left[c_{9}, \gamma_{3}\right]=15, \quad\left[c_{9}, \beta_{4}\right]=9 .
\end{aligned}
$$

The bisecants of $\gamma_{3}$ which meet $\beta_{4}$ lie on $R_{6}: \gamma_{3}^{3}+\beta_{4}$ and have for images in $\left(x^{\prime}\right)$ the points of a curve $\rho_{8}^{\prime}$. The surface $L_{4}^{\prime}$ has 10 singular tangent planes, images of the 6 composite quintics of the web, one of whose components is a quadric through $\gamma_{3}$ and 2 of the points $P$, and the four nodal quintics. Two nodal quintics intersect in a fundamental sextic $d_{6}$ meeting $\gamma_{3}$ in 10 points, $\beta_{4}$ in 6 , and through the four points $P$, and a cubic $t_{i k}$ meeting $\gamma_{3}$ in 5 points, $\beta_{4}$ in 3 , and through 2 of the points $P$. Through each point $P$ passes a fundamental conic $p_{2}$ meeting $\gamma_{3}$ in 3 points, $\beta_{4}$ in 2 points, and a bisecant $g$ of $\gamma_{3}$. The sextic, the 6 cubics, the 4 conics, and the four lines have for images the 15 double points of $L_{4}^{\prime}$. The surface $L_{4}^{\prime}$ is the focal surface of a line congruence of order 2 and class 3 . The curve $\rho_{8}^{\prime}$ is tangent to $L_{4}^{\prime}$ at 16 points $R^{\prime}$, images of the 16 generators $r$ in which $K_{10}$ meets $R_{6}$.

We may now write

$$
\begin{aligned}
& \gamma_{3} \sim \Gamma_{15}^{\prime}: \rho_{8}^{\prime 2}, \quad \beta_{4} \sim B_{9}^{\prime}: \rho_{8}^{\prime}, \\
& s_{1} \sim s_{9}^{\prime}: \rho_{8}^{\prime}, \quad c_{1} \sim c_{5}^{\prime}, \quad\left[c_{5}^{\prime}, \rho_{8}^{\prime}\right]=6, \quad K_{10}: \gamma_{3}^{4}+\beta_{4}^{2} \sim L_{4}^{\prime} .
\end{aligned}
$$

In $I$ the results are

$$
\begin{aligned}
& s_{1} \sim s_{38}: \gamma_{3}^{15}+\beta_{4}^{8}, \quad \gamma_{3} \sim \Gamma_{63}: \gamma_{3}^{25}+\beta_{4}^{13}, \\
& \beta_{4} \sim B_{39}: \gamma_{3}^{15}+\beta_{4}^{9}, \quad \rho_{45} \sim R_{6}: \gamma_{3}^{3}+\beta_{4} \text {. }
\end{aligned}
$$


33. Case D. The basis quartic consists of two lines $\alpha, \bar{\alpha}$ and a conic $\beta_{2}$, each a bisecant of $\gamma_{3}$; the system has three basis points $P$. We have therefore

$$
\begin{gathered}
s_{1}^{\prime} \sim s_{5}: \gamma_{3}^{2}+\alpha+\bar{\alpha}+\beta_{2}+3 P, \\
c_{1}^{\prime} \sim c_{9}, \quad p=1 ; \quad\left[c_{9}, \gamma_{3}\right]=14, \quad\left[c_{9}, \alpha\right]=2, \quad\left[c_{9}, \beta_{2}\right]=8 .
\end{gathered}
$$

The quadric $H_{2}$ through $\gamma_{3}, \alpha$, and $\bar{\alpha}$ with a cubic of the pencil through $\gamma_{3}, \beta_{2}$ and the points $P$ make up a composite quintic of the web. Hence the image of $H_{2}$ is a line $h^{\prime}$ whose image in $(x)$ is $H_{2}$ and the quartic $h_{4}$ common to the pencil of cubics. There are 10 singular tangent planes to $L_{4}^{\prime}$, images of the composite quintics of the web, one of whose components is either a quadric through $\gamma_{3}$ and $\alpha$ or $\bar{\alpha}$ and one point $P$, or the plane of $\beta_{2}$, or a nodal quintic.

The nodal quintics have a common quintic curve, and meet by pairs in one of three quartic curves. Through each point $P$ passes a bisecant $g$ of $\gamma_{3}$. In the plane of $\beta_{2}$ are two lines $t$ meeting $\gamma_{3}$ and $\alpha$ or $\bar{\alpha}$. There are 6 fundamental conics, two through each point $P$, meeting $\gamma_{3} 3$ times, $\alpha, \bar{\alpha}$ and $\beta_{2}$ each once.

The quintic curve, the three quartics, the six conics, and the five lines have for images the 15 double points of $L_{4}^{\prime}$.*

The bisecants of $\gamma_{3}$ which meet $\beta_{2}$ lie on a ruled surface $R_{4}$ having $\gamma_{3}$ double; they have for images the points of a curve $\rho_{6}^{\prime}$ touching $L_{4}^{\prime}$ in 12 points $R^{\prime}$, images of the 12 generators of $R_{4}$ on $K_{10}$. Hence we have

$\alpha \sim A_{2}^{\prime}: h^{\prime}, \quad \bar{\alpha} \sim \bar{A}^{\prime}: h^{\prime}, \quad \beta_{2} \sim B_{8}^{\prime}:{h^{\prime 2}}^{2}+\rho_{6}^{\prime}, \quad \gamma_{3} \sim \Gamma_{14}^{\prime}: h^{\prime 3}+\rho_{6}^{\prime 2}$, $s_{1} \sim s_{9}^{\prime}:{h^{\prime 2}}^{2}+\rho_{6}^{\prime}$,

and in the involution $I$

$$
\begin{array}{cc}
s_{1} \sim s_{36}: \gamma_{3}^{14}+\alpha^{7}+\bar{\alpha}^{7}+\beta_{2}^{8}, & \alpha \sim A_{8}: \gamma_{3}^{3}+\alpha^{2}+\bar{\alpha}+\beta_{2}^{2}, \\
\bar{\alpha} \sim \bar{A}_{8}: \gamma_{3}^{3}+\alpha+\dot{\alpha}^{2}+\beta_{2}^{2}, & \beta_{2} \sim B_{32}: \gamma^{12}+\alpha^{6}+\bar{\alpha}^{6}+\beta_{3}^{8}, \\
\gamma_{3} \sim \Gamma_{56}: \gamma_{3}^{22}+\alpha^{11}+\bar{\alpha}^{11}+\beta_{2}^{12}, \rho_{32} \sim R_{4}: \gamma_{3}^{2}+\beta_{2}, h_{4} \sim H_{2}: \gamma_{3}+\alpha+\bar{\alpha} .
\end{array}
$$

34. Case $\mathbf{E}$. The basis curve is a quintic $\beta_{5}$ of genus 1 , meeting $\gamma_{3}$ in 7 points. There are three points $P$. Here

$$
\begin{aligned}
& s_{1}^{\prime} \sim s_{5}: \gamma_{3}^{2}+\beta_{5}+3 P \\
& c_{1}^{\prime} \sim c_{8}, \quad p=1 ; \quad\left[c_{8}, \gamma_{3}\right]=13, \quad\left[c_{8}, \beta_{5}\right]=9 .
\end{aligned}
$$

* The arrangement of the double points on the singular tangent planes can be determined at once from the preceding discussion of the curves on the composite surfaces of the web. While $L_{4}^{\prime}$ is the same in this as in the preceding case, the distribution of the fundamental curves in $(x)$ is quite different. 
The bisecants of $\gamma_{3}$ which meet $\beta_{5}$ lie on a surface $R_{6}: \gamma_{3}^{3}+\beta_{5}$ and have for images the points of a curve $\rho_{7}^{\prime}$, touching $L_{4}^{\prime}$ in 14 points $R^{\prime}$, images of the 14 generators $r$ of $R_{6}$ lying on $K_{10}$.

The web contains three composite quintics of which one component is a quadric through $\gamma_{3}$ and two of the points $P$; it also contains three nodal quintics. Hence $L_{4}^{\prime}$ has 6 singular tangent planes. There are 3 lines $g$, bisecants of $\gamma_{3}$ through each point $P$; one trisecant $d$ of $\beta_{5}$ meets $\gamma_{3}$. The three nodal quintics have a common quintic curve and meet in pairs in one of three cubics; there are 6 conics meeting $\gamma_{3}$ in 3 points and $\beta_{5}$ in 3 points; two pass through each $P$.

The surface $L_{4}^{\prime}$ has therefore 14 double points and is the focal surface of a line congruence of order 2 and class 4 . We have

$$
s_{1} \sim s_{8}^{\prime}: \rho_{7}^{\prime}, \quad \beta_{6} \sim B_{9}^{\prime}: \rho_{7}^{\prime}, \quad \gamma_{3} \sim \Gamma_{13}^{\prime}: \rho_{7}^{\prime 2},
$$

and in the involution $I$

$$
\begin{array}{ll}
s_{1} \sim s_{33}: \gamma_{3}^{33}+\beta_{5}^{7}, & \beta_{5} \sim B_{39}: \gamma_{3}^{15}+\beta_{5}^{9}, \\
\gamma_{3} \sim \Gamma_{53}: \gamma_{3}^{21}+\beta_{5}^{11}, & \rho_{32} \sim R_{6}: \gamma_{3}^{3}+\beta_{5} .
\end{array}
$$

35. Case F. The quintic basis curve consists of two bisecants $\alpha, \bar{\alpha}$ of $\gamma_{3}$ and a cubic $\beta_{3}$ meeting $\gamma_{3}$ in 4 points; there are 2 basis points $P$. We therefore have

$$
\begin{aligned}
& s_{1}^{\prime} \sim s_{5}: \gamma_{3}^{2}+\alpha+\bar{\alpha}+\beta_{3}+2 P, \\
& c_{1}^{\prime} \sim c_{8}, \quad p=1 ; \quad\left[c_{8}, \gamma_{3}\right]=12, \quad\left[c_{8}, \alpha\right]=2, \quad\left[c_{8}, \beta_{3}\right]=8 .
\end{aligned}
$$

The quadric $H_{2}$ through $\gamma_{3}, \alpha$, and $\bar{\alpha}$ with a cubic of the pencil through $\gamma_{3}, \beta_{3}$ and the points $P$ make up a composite quintic of the web. Hence the image of $H_{2}$ is a line $h^{\prime}$ whose image in $(x)$ is $H_{2}$ and the cubic curve $h_{3}$, common to the pencil of cubic surfaces. There are 6 singular tangent planes to $L_{4}^{\prime}$ images of the four composite quintics of the web; one component is a quadric through $\gamma_{3}, \alpha$ or $\bar{\alpha}$ and one point $P$; the other two images are the nodal quintics. The nodal quintics intersect in 2 fundamental quartics. There is a bisecant $g$ of $\gamma_{3}$ through each point $P$, and there are four lines $d$ meeting $\gamma_{3}, \alpha, \bar{\alpha}$ and $\beta_{3}$.

There are 4 fundamental conics, each passing through one point $P$, meeting $\beta_{3}$ in 3 points, $\gamma_{3}$ in 2 , and meeting each bisecant $\alpha$. Two conics constitute the intersection of two of the cubics in the composite quintics. Finally, there are 2 cubics, each meeting $\gamma_{3}$ in 4 points, $\beta_{3}$ in 4 points, each bisecant in one point, and passing through one point $P$. The 2 quartics, the 2 lines $g$, the 4 lines $d$, the 4 conics and the 2 cubics have for images the 14 double points of $L_{4}^{\prime}$, which is the same surface as in Case $E$. 
The bisecants of $\gamma_{3}$ which meet $\beta_{3}$ lie on a surface $R_{4}: \gamma_{3}^{2}+\beta_{3}$ and have for images the points of a curve $\rho_{b}^{\prime}$ which is tangent to $L_{4}^{\prime}$ at 10 points $R^{\prime}$; these points of contact are images of the 10 generators of $R_{4}$ which lie on $K_{10}$. Hence we may write

$s_{1} \sim s_{8}^{\prime}: h^{\prime 2}+\rho_{5}^{\prime}$,

$\alpha \sim A_{2}^{\prime}: h^{\prime}, \quad \bar{\alpha} \sim \bar{A}_{2}^{\prime}: h^{\prime}, \quad \beta_{3} \sim B_{8}^{\prime}: h^{\prime 2}+\rho_{5}^{\prime}, \quad \gamma_{3} \sim \Gamma_{12}^{\prime}: h^{\prime 3}+\rho_{5}^{\prime}$,

and in the involution $I$

$$
\begin{array}{cc}
s_{1} \sim s_{31}: \gamma_{3}^{12}+\alpha^{6}+\bar{\alpha}^{6}+\beta_{3}^{7}, & \alpha \sim A_{8}: \gamma_{3}^{3}+\alpha^{2}+\bar{\alpha}+\beta_{3}^{2}, \\
\bar{\alpha} \sim \bar{A}_{8}: \gamma_{3}^{3}+\alpha+\bar{\alpha}^{2}+\beta_{3}^{2}, \quad \gamma_{3} \sim \Gamma_{46}: \gamma_{3}^{18}+\alpha^{9}+\bar{\alpha}^{9}+\beta_{3}^{10}, \\
\beta_{3} \sim B_{32}: \gamma_{3}^{12}+\alpha^{6}+\bar{\alpha}^{6}+\beta_{3}^{8}, \quad h_{3} \sim H_{2}: \gamma_{3}+\alpha+\bar{\alpha}, \quad \rho_{21} \sim R_{4}: \gamma_{3}^{3}+\beta_{3} .
\end{array}
$$

36. Case G. Here we have a sextic basis curve $\beta_{6}$ of genus 1 , meeting $\gamma_{3}$ in 9 points; there are 2 basis points $P$. Hence

$$
\begin{aligned}
& s_{1}^{\prime} \sim s_{5}: \gamma_{3}^{2}+\beta_{6}+2 P, \\
& c_{1}^{\prime} \sim c_{7}, \quad p=1 ; \quad\left[c_{7}, \gamma_{3}\right]=11, \quad\left[c_{7}, \beta_{6}\right]=9 .
\end{aligned}
$$

The bisecants of $\gamma_{3}$ which meet $\beta_{6}$ lie on $R_{6}: \gamma_{3}^{3}+\beta_{6}$ and have for images the points of the curve $\rho_{6}^{\prime}$ which is tangent to $L_{4}^{\prime}$ in 12 points $R^{\prime}$, images of the 12 generators of $R_{6}$ on $K_{10}$. The web contains one composite quintic, one component being the quadric through $\gamma_{3}$ and the points $P$; there are two nodal quintics. The surface $L_{4}^{\prime}$ has therefore 3 singular tangent planes. There are 2 lines $g$, bisecants of $\gamma_{3}$, through each point $P$; there are also 3 trisecants of $\beta_{6}$ which meet $\gamma_{3}$.

The two nodal quintics meet in a fundamental quartic and cubic. Through each point $P$ pass three fundamental conics meeting $\gamma_{3}$ and $\beta_{6}$ each in 3 points. Hence the surface $L_{4}^{\prime}$ has 13 double points and is the focal surface of a line congruence of order 2 and class 5 .* $^{*}$

We then have

$$
s_{1} \sim s_{7}^{\prime}: \rho_{6}^{\prime}, \quad \beta_{6} \sim B_{9}^{\prime}: \rho_{6}^{\prime}, \quad \gamma_{3} \sim \Gamma_{11}^{\prime}: \rho_{6}^{\prime 2},
$$

and in the involution $I$

$$
\begin{aligned}
& s_{1} \sim s_{28}: \gamma_{3}^{11}+\beta_{6}^{6}, \quad \gamma_{3} \sim \Gamma_{43}: \gamma_{3}^{17}+\beta_{6}^{9}, \\
& \beta_{6} \sim B_{39}: \gamma_{3}^{15}+\beta_{6}^{9}, \quad \rho_{21} \sim R_{6}: \gamma_{3}^{3}+\beta_{6} .
\end{aligned}
$$

37. Case H. The basis sextic consists of 2 bisecants $\alpha, \bar{\alpha}$ of $\gamma_{3}$ and a quartic $\beta_{4}$ meeting $\gamma_{3}$ in 6 points; there is one point $P$. Hence

$$
\begin{aligned}
& s_{1}^{\prime} \sim s_{5}: \gamma_{3}^{2}+\alpha+\bar{\alpha}+\beta_{4}+P, \\
& c_{1}^{\prime} \sim c_{7}, \quad p=1 ; \quad\left[c_{7}, \gamma_{3}\right]=10, \quad\left[c_{7}, \alpha\right]=2, \quad\left[c_{7}, \beta_{4}\right]=8 .
\end{aligned}
$$

\footnotetext{
* Kummer, 1. c., pp. 88-94.
} 
The bisecants of $\gamma_{3}$ which meet $\beta_{4}$ lie on a surface $R_{4}: \gamma_{3}^{2}+\beta_{4}$ and have for images the points of a curve $\rho_{4}^{\prime}$ which touches $L_{4}^{\prime}$ in 8 points $R^{\prime}$; the points of contact are the images of the 8 generators of $R_{4}$ which lie on $K_{10}$. The quadric $H_{2}: \gamma_{3}+\alpha+\bar{\alpha}$ and a cubic surface of the pencil through $\gamma_{3}, \beta_{4}$, and $P$ make up a composite quintic of the web. Hence the image of $\mathrm{H}_{2}$ is a line $h^{\prime}$ whose image in $(x)$ is $H_{2}$ and the conic $h_{2}$ common to the cubics of the pencil.

There are 2 composite quintics with one component a quadric through $\gamma_{3}$, $\alpha$ or $\bar{\alpha}$ and $P$; there is one nodal quintic. The line $g$ is the bisecant of $\gamma_{3}$ through $P$. Three lines meet $\beta_{4}$ twice and meet $\gamma_{3}$ and $\alpha$ or $\bar{\alpha}$; the composite quintics intersect each other and each intersects the nodal quintic in a fundamental conic; two of the conics pass through $P$, meet $\beta_{4}$ in 2 points, $\alpha_{3}$ in 3 points, and $\alpha$ or $\bar{\alpha}$ in one; the third meets $\alpha$ and $\bar{\alpha}, \beta_{4}$ in 2 points, and $\gamma_{3}$ in 3 points. There are 3 fundamental cubics meeting $\alpha, \bar{\alpha}, \gamma_{3}$ in 4 points, $\beta_{4}$ in 4 and passing through $P$.

The surface $L_{4}^{\prime}$ has the same form as in the last preceding case.

We have then

$s_{1} \sim s_{7}^{\prime}: h^{\prime 2}+\rho_{4}^{\prime}, \quad \gamma_{3} \sim \Gamma_{10}^{\prime}: h^{\prime 2}+{\rho_{4}^{\prime 2}}^{2} \quad \alpha \sim A_{2}^{\prime}: h^{\prime}, \quad \beta_{4} \sim B_{8}^{\prime}: \rho_{4}^{\prime}$, and in the involution $I$

$$
\begin{array}{cc}
s_{1} \sim s_{26}: \gamma_{3}^{10}+\alpha^{5}+\bar{\alpha}^{5}+\beta_{4}^{6}, & \gamma_{3} \sim \Gamma_{36}: \gamma_{3}^{14}+\alpha^{7}+\bar{\alpha}^{7}+\beta_{4}^{8}, \\
\alpha \sim A_{8}: \gamma_{3}^{8}+\alpha^{2}+\bar{\alpha}+\beta_{4}^{2}, \quad \bar{\alpha} \sim \bar{A}_{8}: \gamma_{3}^{3}+\alpha+\bar{\alpha}^{2}+\beta_{4}^{2}, \\
\beta_{4} \sim B_{32}: \gamma_{3}^{12}+\alpha^{8}+\bar{\alpha}^{8}+\beta_{4}^{8}, \quad h_{2} \sim H_{2}: \gamma_{3}+\alpha+\bar{\alpha}, \quad \rho_{12} \sim R_{4}: \gamma_{3}^{2}+\beta_{4} .
\end{array}
$$

38. Case $I$. The basis curve is a $\beta_{7}$ of genus 1 , with no five-fold secants, meeting $\gamma_{3}$ in 11 points; there is one basis point $P$. Here

$$
\begin{aligned}
s_{1}^{\prime} & \sim s_{5}: \gamma_{3}^{2}+\beta_{7}+P, \\
c_{1}^{\prime} & \sim c_{6}, \quad p=1 ; \quad\left[c_{6}, \gamma_{3}\right]=9, \quad\left[c_{6}, \beta_{7}\right]=9 .
\end{aligned}
$$

The bisecants of $\gamma_{3}$ which meet $\beta_{7}$ lie on a surface $R_{6}: \gamma_{3}^{3}+\beta_{7}$ and have for images the points of a curve $\rho_{5}^{\prime}$, tangent to $L_{4}^{\prime}$ at 10 points, images of the 10 generators $r$ common to $R_{6}$ and $K_{10}$.

There is one singular tangent plane of $L_{4}$, image of the nodal quintic of the web. There is one line $g$, bisecant of $\gamma_{3}$ from $P$, and 6 lines $d$, trisecants of $\beta_{7}$ meeting $\gamma_{3}$, also 4 fundamental conics and one cubic. Hence $L_{4}^{\prime}$ has 12 double points. It is the focal surface of a line congruence of order 2 and class $6 .^{*}$

Hence

$$
s_{1} \sim s_{6}^{\prime}: \rho_{5}^{\prime}, \quad \gamma_{3} \sim \Gamma_{9}^{\prime}: \rho_{5}^{\prime 2}, \quad \beta_{7} \sim B_{9}^{\prime}: \mu_{5}^{\prime},
$$

* Kummer, l. c., pn. 102-107. 
and in the involution $I$

$$
\begin{aligned}
& s_{1} \sim s_{23}: \gamma_{3}^{9}+\beta_{7}^{5}, \quad \gamma_{3} \sim \Gamma_{33}: \gamma_{3}^{13}+\beta_{7}^{7}, \\
& \beta_{7} \sim B_{39}: \gamma_{3}^{15}+\beta_{7}^{9}, \quad \rho_{12} \sim R_{6}: \gamma_{3}^{3}+\beta_{7} *^{*}
\end{aligned}
$$

39. Case J. The basis curve consists of 2 bisecants $\alpha, \bar{\alpha}$ of $\gamma_{3}$ and a rational quintic $\beta_{5}$ meeting $\gamma_{3}$ in 8 points, hence

$$
\begin{aligned}
& s_{1}^{\prime} \sim s_{5}: \gamma_{3}^{2}+\alpha+\bar{\alpha}+\beta_{5}, \\
& c_{1}^{\prime} \sim c_{6}, \quad p=1 ; \quad\left[c_{6}, \gamma_{3}\right]=8, \quad\left[c_{6}, \alpha\right]=2, \quad\left[c_{6}, \beta_{5}\right]=8 .
\end{aligned}
$$

The quadric $H_{2}: \gamma_{3}+\alpha+\bar{\alpha}$ and a cubic of the pencil through $\beta_{5}$ and $\gamma_{3}$ make up a composite quintic of the web. Hence the image of $H_{2}$ is a line $h^{\prime}$, whose complete image in $(x)$ consists of $H_{2}$ and the line $h$, common to the cubics of the pencil. The bisecants of $\gamma_{3}$ which meet $\beta_{5}$ lie on a surface $R_{4}: \gamma_{3}^{2}+\beta_{5}$ and have for images the points of a cubic $\rho_{3}^{\prime}$ tangent to $L_{4}^{\prime}$ at 6 points, images of the 6 generators $r$ common to $R_{4}$ and to $K_{10}$. We have therefore

$$
\begin{aligned}
& s_{1} \sim s_{6}^{\prime}: h^{\prime 2}+\rho_{3}^{\prime}, \quad \gamma_{3} \sim \Gamma_{8}^{\prime}: h^{\prime 3}+\rho_{3}^{\prime 2}, \\
& \alpha \sim A_{2}^{1}: h^{\prime}, \quad \beta_{5} \sim B_{8}^{\prime}: h^{\prime 2}+\rho_{3}^{\prime},
\end{aligned}
$$

and in the involution $I$

$$
\begin{array}{cc}
s_{1} \sim s_{21}: \gamma_{3}^{8}+\alpha^{4}+\bar{\alpha}^{4}+\beta_{5}^{5}, & \gamma_{3} \sim \Gamma_{20}: \gamma_{3}^{10}+\alpha^{5}+\bar{\alpha}^{5}+\beta_{5}^{6}, \\
\beta_{5} \sim B_{32}: \gamma_{3}^{13}+\alpha^{6}+\bar{\alpha}^{6}+\beta_{5}^{8}, & \alpha \sim A_{8}: \gamma_{3}^{3}+\alpha^{2}+\bar{\alpha}^{2}+\beta_{5}^{2}, \\
\rho_{5} \sim R_{4}: \gamma_{3}^{3}+\beta_{5}, & h \sim H_{2}: \gamma_{3}+\alpha+\bar{\alpha} .
\end{array}
$$

There are 8 fundamental lines and 4 fundamental conics. The surface $L_{4}^{\prime}$ is the focal surface of a line congruence of order 2 and class 6 , without singular planes. $f$

40. Quintics with a double quartic curve. The curve $\gamma_{4}$ must be of genus 1 . By Art. 16 we have

$$
p^{\prime}+3 s=6 m^{\prime}-2, \quad \xi+s=m^{\prime}+5,
$$

the possible solutions of which are

$\begin{array}{rrrr}m^{\prime} & p^{\prime} & 8 & \xi \\ A \ldots \ldots 3 & -2 & 6 & 2 \\ B \ldots \ldots 4 & -2 & 8 & 1 \\ C \ldots \ldots 5 & -2 & 10 & 0\end{array}$

* If $\beta_{7}$ lies on a cubic surface (Noether, 1. c., p. 91), this surface contains $\gamma_{3}$ and there is a pencil of composite quintics. The corresponding involution differs somewhat from the type just obtained.

† Kummer, l. e., pp. 94-102. 
41. Case A. The simple basis curve consists of three bisecants of $\gamma_{4}$; there are 2 basis points $P$. By means of a cubic transformation having $\gamma_{4}+2 \alpha$ for a basis sextic of genus 3 , the web of quintics can be transformed into a web of cubics having a quartic curve of genus 1 , a bisecant, and 2 basis points for basis elements. The quartic and its bisecant constitute a quintic of genus 2 , hence this is included as a particular case of that discussed in Article 9.

42. Case B. By proceeding as in the last preceding case, this is at once reducible to that of Article 10.

43. Case C. The simple basis curve consists of a bisecant $\alpha$ of $\gamma_{4}$ and of 2 conics $\beta_{2}, \bar{\beta}_{2}$ each meeting $\gamma_{4}$ in 4 points. We have therefore

$$
\begin{aligned}
& s_{1}^{\prime} \sim s_{5}: \gamma_{4}^{2}+\alpha+\beta_{2}+\bar{\beta}_{2}, \\
& c_{1}^{\prime} \sim c_{4}, \quad p=1 ; \quad\left[c_{4}, \alpha\right]=2, \quad\left[c_{4}, \gamma_{4}\right]=6, \quad\left[c_{4}, \beta_{2}\right]=2 .
\end{aligned}
$$

The quadric $R_{2}: \gamma_{4}+\alpha$ and a cubic of the pencil through $\gamma_{4}, \beta_{2}$ and $\bar{\beta}_{2}$ make up a composite quintic of the web. The image of $R_{2}$ is therefore a line $\rho^{\prime}$ having for image in $(x)$ the quadric $R_{2}$ and the line $\rho$ common to the cubics of the pencil. The quadric $H_{2}: \gamma_{4}+\beta_{2}$ and a cubic of the bundle through $\gamma_{4}, \bar{\beta}_{2}$, and $\alpha$ make up a composite quintic of the web. The image of $H_{2}$ is therefore a point $P^{\prime}$. Similarly, the image of $\bar{H}_{2}: \gamma_{4}+\bar{\beta}_{2}$ is a point $\bar{P}^{\prime}$. We have therefore

$s_{1} \sim s_{4}^{\prime}: \rho^{\prime}+P^{\prime}+\bar{P}^{\prime}, \quad \alpha \sim A_{2}^{\prime}: \rho^{\prime}, \quad \beta_{2} \sim B_{2}^{\prime}: P^{\prime}, \quad \bar{\beta}_{2} \sim \bar{B}_{2}^{\prime}: \bar{P}^{\prime}$, $\gamma_{4} \sim \Gamma_{6}:{\rho^{\prime 2}}^{2}+{P^{\prime 2}}^{2} \bar{P}^{\prime 2}$,

and in the involution $I$

$$
\begin{aligned}
& s_{1} \sim s_{13}: \gamma_{4}^{5}+\alpha^{3}+\beta_{2}^{3}+\bar{\beta}_{2}^{3}, \quad \alpha \sim A_{8}: \gamma_{4}^{3}+\alpha^{2}+\beta_{2}^{2}+\bar{\beta}_{2}^{2}, \\
& \beta_{2} \sim B_{8}: \gamma_{4}^{3}+\alpha^{2}+\beta_{2}^{2}+\bar{\beta}_{2}^{2}, \quad \bar{\beta}_{2} \sim \bar{B}_{8}: \gamma_{4}^{3}+\alpha^{2}+\beta_{2}^{2}+\bar{\beta}_{2}^{2}, \\
& \gamma_{4} \sim \Gamma_{18}: \gamma_{4}^{7}+\alpha^{4}+\beta_{2}^{4}+\bar{\beta}_{2}^{4} .
\end{aligned}
$$

The line $\rho^{\prime}$ is tangent to $L_{4}^{\prime}$ at 2 points $R^{\prime}$, images of the 2 generators of $R_{2}$ on $K_{10}$. There are 8 fundamental lines.

44. Conclusion. This completes the consideration of involutions derivable from $(2,1)$ correspondences using webs of surfaces of order not greater than 5 . When surfaces of higher order are used, all the involutions associated with $L^{\prime}$ of order 4 are reducible to some type obtained in this paper.

Cornell University 